Para enlazar con este artículo / To link to this article:

http://dx.doi.org/10.14198/fem.2017.29.10

Para citar este artículo / To cite this article:

Navarro-Astor, Elena, Arianna Guardiola-Víllora y Luisa Basset-Salom. «El profesorado de la Universitat Politècnica de València desde la perspectiva de género». En Marcos Jesús Iglesias Martínez e Inés Lozano Cabezas (coords.), La (in)visibilidad de las mujeres en la Educación Superior: retos y desafios en la Academia. Feminismo/s, 29 (junio 2017): 243-277, DOI: 10.14198/fem.2017.29.10

\title{
EL PROFESORADO DE LA UNIVERSITAT POLITĖCNICA DE VALÈNCIA DESDE LA PERSPECTIVA DE GÉNERO
}

\author{
UNIVERSITAT POLITÈCNICA DE VALÈNCIA'S FACULTY \\ FROM A GENDER PERSPECTIVE
}

\author{
Elena NAVARRO-ASTOR \\ enavarro@upvnet.upv.es \\ orcid.org/0000-0002-1588-9186 \\ Arianna GUARDiOLA-VÍLlORA \\ aguardio@mes.upv.es \\ orcid.org/0000-0003-3234-0547 \\ Luisa BASSET-SALOM \\ lbasset@mes.upv.es \\ orcid.org/0000-0002-3563-7963 \\ Universitat Politècnica de València
}

\section{Resumen}

Medir es conocer: El diagnóstico es un paso imprescindible para la resolución de cualquier problema. En este documento se analiza la situación del profesorado de la UPV, con objeto de determinar el grado de masculinización del Personal Docente e Investigador de sus escuelas y departamentos. Esta descripción cuantitativa de la situación de facto en el momento del estudio muestra el desequilibrio existente en la institución: además de una segregación horizontal, es evidente el importante grado de segregación vertical. La drástica disminución de la proporción de mujeres a medida que se asciende en la jerarquía académica hace que su presencia en alguna de las categorías superiores sea anecdótica. Es indiscutible la existencia del techo de cristal en la carrera docente de las profesoras de la UPV. Este estudio constituye un paso previo para el futuro desarrollo de estrategias, programas y planes necesarios para alcanzar una composición equilibrada de la plantilla docente. 
Palabras clave: desigualdad de género; Educación Superior; estadísticas; profesorado; Universitat Politècnica de València.

\begin{abstract}
To measure is to know: The diagnosis is a prerequisite to solve any problem. In this paper the situation of the UPV's faculty is analized in order to establish the degree of masculinisation of the teaching and research staff in its schools and departments. This quantitative description of the «de facto» situation at the time of the study shows the current imbalance at the institution: in addition to horizontal segregation, significant vertical segregation is evident. The drastic decline in women's proportion as one moves up to the academic hierarchy makes incidental their presence in some of the higher academic ranks. The existence of the glass ceiling in women's academic careers at the UPV is undeniable. This study constitutes a first step for the future development of strategies, programmes and plans to achieve a balanced composition of its faculty.
\end{abstract}

Keywords: faculty; gender inequality; higher education; statistics; Universitat Politècnica de València. 


\section{INTRODUCCIÓN}

En las últimas décadas ha aumentado la conciencia de las sociedades desarrolladas acerca de la igualdad de trato y de oportunidades entre hombres y mujeres, implementándose políticas de género en los diferentes ámbitos de la sociedad (Pastor et al. 2015).

En el ámbito de la Educación Superior hay, por un lado, señales de progreso hacia la igualdad de género entre los estudiantes. Así, en la UE las mujeres representaron de media el $47 \%$ de los estudiantes de tercer ciclo y su participación ha aumentado a mayor velocidad que la de los hombres desde 2003 (European Comission 2016). No obstante, los datos muestran que todavía existen diferencias importantes en función de las disciplinas estudiadas: ingeniería, producción y construcción siguen estando muy masculinizadas, con medias para la UE de $28 \%$ de alumnas de tercer ciclo. Para el caso de informática, su participación se reduce al 21\% (European Comission 2016). En España, «un siglo de historia universitaria ha sido necesario para que el número de estudiantes universitarias sea superior al de sus iguales masculinos» (Lozano Cabezas et al. 2013). En el curso 2013-14 las mujeres matriculadas en los estudios de grado fueron mayoría (54,4\%), al igual que las egresadas (57,3\%) (MECyD 2015a). Estos datos son similares a los de años precedentes, y también la distribución por áreas de conocimiento, con una proporción de hombres muy superior a la de mujeres en ingeniería y arquitectura (74,1\%) (MECyD 2015a).

Por otro lado, las mujeres están sub representadas en los estratos más altos de la jerarquía académica, es decir, existe segregación vertical (European Comission 2016, Lozano Cabezas et al. 2013 y 2016, Guil y Flecha, Pastor et al. 2015, Sánchez de Madariaga 2013, Guil 2008). Así, la representación de las mujeres entre el Personal Docente e Investigador (PDI) de las universidades públicas españolas se reduce al 39,4\%, y a tan solo 20,7\% para el caso de las funcionarias Catedráticas de Universidad (CU) (MECyD, 2015a). En la comparación internacional, España está ligeramente por debajo del promedio de la UE-27 en cuanto a proporción de mujeres CU y eméritas (MEC-UMYC, 2014). Si se analizan los datos por área de conocimiento se observa que las mujeres todavía tienen menor presencia en ingeniería y arquitectura (20,6\% 
del PDI, 15,9\% de los CU). De ahí que se afirme que para el caso del sistema universitario español, el diagnóstico elaborado hace 13 años seguiría siendo apropiado (Matus-López y Gallego-Morón), mostrando la lentitud del cambio.

En este contexto, diversos organismos internacionales señalan la necesidad de promover la integración de las mujeres en la ciencia para defender sus derechos y también para evitar el mal uso de los recursos (Mauleón y Bordons). Así, la Comisión Europea se manifiesta seriamente comprometida con la educación, defendiendo la construcción de un modelo de ciudadanía basado en la igualdad de género (Commission of the European Communities). En su informe de 2012 (European Commission 2012) planteó líneas de acción concretas para poner en práctica una política de género que supusiera un cambio estructural de las universidades europeas. Entre otras medidas, dicho informe recomienda llevar a cabo acciones de sensibilización y concienciación inicial, la construcción de ambientes positivos, el desarrollo de indicadores y estadísticas que contribuyan a visualizar la situación o la financiación de programas y proyectos específicos. Pero para implementar estas estrategias cada universidad debe partir del conocimiento y diagnóstico del propio entorno institucional, además de contar con el apoyo incondicional de su gobierno (Lozano Cabezas et al. 2013). Además, las instituciones europeas han incluido el género como un tema prioritario y transversal en el programa marco de investigación e innovación, Horizonte 2020 (Pastor et al. 2015; MEC-UMYC; Sánchez de Madariaga 2013).

En España, la Ley Orgánica 3/2007, de 22 de marzo, para la igualdad efectiva de mujeres y hombres ha trazado el camino a seguir para alcanzar la igualdad de género, incluyendo la obligación de negociar planes de igualdad en las empresas de más de 250 empleados, previo diagnóstico integral de equidad (Acosta et al.; Marqués et al.; Elizondo et al. 2010). Asimismo, la Ley Orgánica 4/2007 de universidades establece diferentes medidas con el fin de alcanzar la paridad en los órganos representativos y de gobierno en las universidades, y para que las mujeres mejoren su representación en los grupos de investigación y en los puestos más altos (Pastor et al. 2014 y 2015). Además, establece la creación de unidades de igualdad de género, con presupuesto propio, personal técnico especializado en temas de género, y personal administrativo.

Así, en la actualidad, las unidades de igualdad y los planes de igualdad son instrumentos clave para que las instituciones de Educación Superior españolas aborden sus objetivos de igualdad de género (Pastor et al. 2015). No obstante, diferencias relativas a financiación, antigüedad, dimensión, rasgos socio-laborales, presencia de grupos de estudios de género, participación en redes de igualdad, entre otras, influyen en el diseño y puesta en práctica de las políticas de igualdad en cada universidad (Acosta et al.; Pastor y Acosta). 
De hecho, en la actualidad sólo 33 de las 52 universidades públicas españolas tienen un plan de igualdad (Pastor et al. 2015).

\section{ANTECEDENTES Y OBJETIVOS}

Las investigaciones sobre la desigualdad de género en Educación Superior tienen una amplia trayectoria en España, siendo en su mayoría estudios de casos de universidades concretas (Matus-López y Gallego-Morón) que describen exhaustivamente la composición demográfica de los colectivos de integrantes de la institución (estudiantes, académicos o personal administrativo) a través de estadísticas. Estos estudios confirman la persistencia de desigualdades de género, evidenciando la existencia de segregación tanto horizontal (concentración de mujeres en determinadas áreas temáticas) como vertical (menor presencia femenina al ascender en la jerarquía profesional (Mauleón y Bordons). Además, estos análisis con perspectiva de género se llevan a cabo para distintas áreas de conocimiento y titulaciones, mostrando importantes diferencias entre ellas.

Destacan por ejemplo el caso de la Universidad de Sevilla (Guil, 2005), de Valladolid (Anguita), de Málaga (Morales et al.), de Alicante (Antón, Lozano Cabezas et al. 2013), de La Laguna (Jiménez), de Castilla y León (Lecuona), Universitat Autónoma de Barcelona (Izquierdo), Universitat Jaume I en Castellón (Alcañiz), Rovira i Virgili en Tarragona (Pastor-Gosálbez et al.), Universitat de València (Barberá et al., Escolano 2002, Fernández-Coronado y González, Perelló 2012 y 2013) y Universidad Politécnica de Madrid (Sánchez de Madariaga 2014). Asimismo se han llevado a cabo estudios comparativos entre distintas universidades como el de Ion et al. y Tomás et al. que comparan la Universidad de Sevilla con la Universidad Autónoma de Barcelona, el de cuatro universidades catalanas (Tomás y Guillamón) y cinco universidades públicas valencianas (Escolano 2006).

El origen de la UPV se sitúa en el curso 1968-1969 cuando se crea el Instituto Politécnico Superior de Valencia que engloba cuatro centros: la Escuela Técnica Superior de Ingenieros Agrónomos, creada en 1959; la Escuela Técnica Superior de Arquitectura, constituida en 1966; la Escuela Técnica Superior de Ingenieros de Caminos, Canales y Puertos, y la Escuela Técnica Superior de Ingenieros Industriales, ambas de 1968. El máximo rango de Universidad llega en 1971. A partir de ese año se van adhiriendo el resto de centros (Marqués et al.; UPV).

En la actualidad, más de 42.000 miembros pertenecen a la UPV, de los cuales en torno a 37.800 son alumnos, 2.600 PDI y 1.700 personal de la administración y los servicios. Está compuesta por 15 centros universitarios, de 
los que 9 son escuelas técnicas superiores, 2 son facultades y 2 son escuelas politécnicas superiores. Asimismo, cuenta con 3 centros adscritos. La UPV ofrece 35 grados relacionados con diferentes ramas de conocimiento: 1) Arte y Humanidades, 2) Ciencias, 3) Ciencias sociales y jurídicas y 4) Ingeniería y Arquitectura, que es la más representativa, puesto que engloba el $66 \%$ de los grados ofrecidos.

Las primeras referencias a la desigualdad de género en las aulas de la UPV aparecen en forma de anecdotario en el libro «Nacimiento de una universidad, algunos recuerdos» del profesor Santos (1993), Secretario General de la Universidad durante más de una década. En él se indica que en el curso 198081, en Arquitectura Técnica la proporción entre los estudiantes matriculados era de 116 alumnas frente a 892 alumnos, finalizando la carrera 12 mujeres y 100 hombres. En cuanto al profesorado de dicha escuela, se indica que había sólo 2 mujeres entre los 54 docentes.

La UPV ha formado parte de trabajos de carácter amplio sobre el tema de la igualdad de género, participando junto a otras universidades. Así, por ejemplo, en un ranking de igualdad de un total de 70 universidades españolas, la UPV se sitúa casi en el extremo final, ocupando el puesto n. ${ }^{\circ} 65$ (Elizondo et al. 2007), pero por delante de la Politécnica de Cartagena, la Politécnica de Catalunya (UPCT) y la Politécnica de Madrid. También ha sido objeto de estudio para una comparativa de la carrera académica del profesorado en las cuatro universidades politécnicas españolas (Marqués et al.). Esta comparativa coloca a la UPV como la Politécnica con mayor porcentaje de mujeres en su PDI en 2012 (29\%, frente al 19\% en la UPCT). Sin embargo, según Escolano (2006), en 2001 la UPV estaba a la cola del resto de universidades públicas valencianas en cuanto a participación de la mujer, puesto que sólo representaban el 22.3\% de los docentes, frente a la media del $30.9 \%$. La distancia entre sexos era todavía mayor en las áreas de conocimiento con mayor nivel de experimentación y sectores técnicos o tecnológicos. En consecuencia, en muchos departamentos de la UPV la presencia de la mujer era «meramente simbólica» (Escolano 2006, 425). De hecho, $71.5 \%$ de sus departamentos tenían menos del $25 \%$ de profesoras y en 3 departamentos no había ninguna mujer (Ingeniería e Infraestructura de los Transportes, Máquinas y Motores Térmicos y Mecanización y Tecnología Agraria). Por el contrario, sólo 3 departamentos tenían mayoría de mujeres, pero ninguno perteneciente al campo de la Ingeniería y la Arquitectura, sino a Humanidades y Ciencias Básicas.

Hasta la fecha únicamente Basset-Salom et al., Guardiola-Víllora et al. y Estellés-Miguel et al. han abordado la descripción de aspectos relacionados con la desigualdad de género en la UPV. Las primeras estudiaron el caso de la 
Escuela Técnica Superior de Arquitectura y el Departamento de Estructuras desde una perspectiva de género, constatando un incremento lento y moderado en la presencia de mujeres entre el profesorado para el periodo 1987-2008, a pesar de que el porcentaje de egresadas iguala al de egresados desde el año 2000. Las segundas analizan la presencia femenina en los equipos de gestión de la institución (departamentos y escuelas), subrayando que es escasa, y que el cargo de secretario/a es el más feminizado. Por último, Estellés-Miguel et al. estudian la estructura y la distribución por género del PAS (Personal de Administración y Servicios).

La UPV, a través del Vicerrectorado de Asuntos Sociales y Responsabilidad Social Corporativa, publicó en 2011 un documento previo a la constitución de su unidad de igualdad (Santamarina et al.) en el que se describe su retraso respecto al resto de universidades valencianas y de universidades politécnicas españolas en la aprobación e implantación de los planes de igualdad. Se indica que «en marzo de 2008 se realizó un estudio diagnóstico de la presencia por género en las distintas categorías profesionales que integran las plantillas de la UPV, en puestos de responsabilidad y en el alumnado, donde se presenta la evolución académica y profesional de las mujeres de la UPV» (Santamarina et al. 15), no habiendo sido posible acceder a dicho estudio.

El primer plan de igualdad de la UPV fue finalmente aprobado el 17 de enero de 2014, diez años después de algunas universidades públicas catalanas, pioneras en su implantación (Santamarina et al.). Pero tampoco en este plan se recogen datos estadísticos sobre la desigualdad horizontal y vertical segmentados por género en los distintos colectivos de la comunidad universitaria. El Plan sí describe las acciones que deben ser implementadas para alcanzar la igualdad, sin embargo, no se determinan quiénes son las entidades o personas responsables de llevarlas a cabo, y tampoco los recursos humanos y económicos destinados a ellas. Según De los Cobos, esto mediría el grado de compromiso institucional con la igualdad.

Hasta la fecha, en España ha habido escasa conciencia sobre la importancia de desagregar los datos por sexo para conocer realmente la situación de las mujeres (Guil y Flecha). Sin embargo, como indica Carmen Vela, Secretaria de Estado de Investigación, Desarrollo e Innovación, «las cifras nos ayudan a definir, ejecutar y evaluar las políticas» (MEC-UMYC 4). Uno de los factores que contribuyen a la activación de medidas destinadas a la igualdad es la visibilización de la distancia de género a partir de la publicación de datos estadísticos. Estos datos son esenciales para realizar un seguimiento longitudinal, analizar la eficacia de las estrategias implementadas y comparar regiones y países (Mauleón y Bordons). Según Elizondo et al. (2010), la mayoría de 
universidades con unidades de igualdad ya cuenta con diagnósticos publicados, no siento éste el caso de la UPV.

En consecuencia, este estudio vendría a cubrir un vacío investigador, puesto que pretende sacar a la luz cuál es la situación de igualdad de género en la UPV. El conocimiento del propio entorno institucional es la base para empezar a cambiar las cosas, el punto de partida para desarrollar el resto de estrategias. Como señalaba Alonso hace más de una década: «las posibilidades de que esta situación de flagrante desigualdad evolucione, dependerá en buena medida de que se comience siquiera a percibirla y reconocerla» (Alonso 616).

Conocidos estos antecedentes, este trabajo responde a la necesidad de afrontar un desafío contemporáneo e histórico a la vez para las instituciones de Educación Superior: las asimetrías de género. De ahí el propósito principal de documentar y visibilizar de forma exhaustiva por primera vez las diferencias de género en la carrera académica del PDI de la UPV: Este objetivo se concreta en los siguientes sub-objetivos:

Examinar la segregación vertical o posición diferencial en la jerarquía del PDI en base a las categorías profesionales (profesorado de los cuerpos docentes universitarios y profesorado contratado laboral).

Analizar la segregación horizontal por sexo, es decir, la concentración de hombres o de mujeres en cada uno de los centros docentes y de los departamentos.

\section{MATERIAL Y MÉTODOS}

Para este estudio se han utilizado los datos recogidos en el directorio del personal de la UPV, accesible desde la intranet, asumiendo que todos los miembros de la comunidad universitaria están incluidos en el mismo. La información disponible (Diciembre 2015) incluye el nombre, categoría, departamento y centro docente al que se encuentra adscrito el PDI.

Los centros docentes que forman parte de la UPV son las 9 Escuelas Técnicas, 2 facultades y 2 escuelas politécnicas superiores de la tabla 1. Los nombres de los 42 departamentos con sus siglas se recogen en la tabla 2. 
Tabla 1. Centros docentes de la UPV (2016)

\begin{tabular}{|l|c|l|l|}
\hline E.T.S. $^{1}$ de Arquitectura & ETSA & E.T.S. de Ingenieros Industriales & ETSII \\
\hline $\begin{array}{l}\text { E.T.S. de Ingeniería } \\
\text { Agronómica y del Medio } \\
\text { Natural }\end{array}$ & ETSIAMN & $\begin{array}{l}\text { E.T.S. de Ingenieros de } \\
\text { Telecomunicación }\end{array}$ & ETSIT \\
\hline E.T.S. de Ingeniería del Diseño & ETSID & $\begin{array}{l}\text { Facultad de Administración y } \\
\text { Dirección de Empresas }\end{array}$ & FADE \\
\hline $\begin{array}{l}\text { E.T.S. de Ingeniería de } \\
\text { Edificación }\end{array}$ & ESTIE & Facultad de Bellas Artes & FBBAA \\
\hline $\begin{array}{l}\text { E.T.S. de Ingeniería Geodésica, } \\
\text { Cartográfica y Topográfica }\end{array}$ & ETSIGCT & $\begin{array}{l}\text { Escuela Politécnica Superior de } \\
\text { Alcoy }\end{array}$ & EPSA \\
\hline $\begin{array}{l}\text { E.T.S. de Ingeniería } \\
\text { Informática }\end{array}$ & ETSINF & $\begin{array}{l}\text { Escuela Politécnica Superior de } \\
\text { Gandía }\end{array}$ & EPSG \\
\hline $\begin{array}{l}\text { E.T.S. de Ingenieros de } \\
\text { Caminos, Canales y Puertos }\end{array}$ & ETSCCP & & \\
\hline
\end{tabular}

${ }^{1}$ E.T.S. son las siglas de Escuela Técnica Superior

Tabla 2. Departamentos de la UPV (2016)

\begin{tabular}{|c|c|c|c|}
\hline D. Biotecnología & BTC & $\begin{array}{l}\text { D. Comun. Audiovisual, } \\
\text { Documentación e H. }{ }^{a} \text { del Arte }\end{array}$ & HAR \\
\hline $\begin{array}{l}\text { D. Ingeniería Cartográfica } \\
\text { Geodesia y Fotogrametría }\end{array}$ & CGF & $\begin{array}{l}\text { D. Ingeniería Hidráulica y Medio } \\
\text { Ambiente }\end{array}$ & HMA \\
\hline D. Composición Arquitectónica & $\mathrm{CPA}$ & D. Lingüística Aplicada & IDM \\
\hline $\begin{array}{l}\text { D. Conservación y Restauración de } \\
\text { Bienes Culturales }\end{array}$ & CRBC & D. Ingeniería Química y Nuclear & IQN \\
\hline D. Construcciones Arquitectónicas & CSA & $\begin{array}{l}\text { D. Ingeniería Rural y } \\
\text { Agroalimentaria }\end{array}$ & IRA \\
\hline $\begin{array}{l}\text { D. Ingeniería de la Construcción y } \\
\text { de Proyectos de Ingeniería Civil }\end{array}$ & CST & $\begin{array}{l}\text { D. Ingeniería de Sistemas y } \\
\text { Automática }\end{array}$ & ISA \\
\hline D. Ciencia Animal & DCA & D. Matemática Aplicada & MAT \\
\hline D. Comunicaciones & DCOM & $\begin{array}{l}\text { D. Ingeniería Mecánica y de } \\
\text { Materiales }\end{array}$ & $\mathrm{MCM}$ \\
\hline D. Economía y Ciencias Sociales & DECS & $\begin{array}{l}\text { D. Mecánica de los Medios } \\
\text { Continuos y Teoría de } \\
\text { Estructuras }\end{array}$ & MES \\
\hline D. Ingeniería Gráfica & DEGI & $\begin{array}{l}\text { D. Máquinas y Motores } \\
\text { Térmicos }\end{array}$ & MOT \\
\hline D. Dibujo & DIB & D. Organización de Empresas & OMP \\
\hline D. Ingeniería Eléctrica & DIE & D. Pintura & PIN \\
\hline
\end{tabular}




\begin{tabular}{|l|c|l|c|}
\hline $\begin{array}{l}\text { D. Informática de Sistemas y } \\
\text { Computadores }\end{array}$ & DISCA & Dpto. proyectos arquitectónicos & PRA \\
\hline D. Proyectos de Ingeniería & DPI & Dpto. producción vegetal & PRV \\
\hline $\begin{array}{l}\text { D. Sistemas Informáticos y } \\
\text { Computación }\end{array}$ & DSIC & D. Química & QIM \\
\hline D. Ecosistemas Agroforestales & EAF & D. Tecnología de Alimentos & TAL \\
\hline $\begin{array}{l}\text { D. Expresión gráfica } \\
\text { Arquitectónica }\end{array}$ & EGA & D. Termodinámica Aplicada & TER \\
\hline $\begin{array}{l}\text { D. Estadística e Investigación } \\
\text { Operativa Aplicadas y Calidad }\end{array}$ & EIO & $\begin{array}{l}\text { D. Ingeniería e Infraestructura } \\
\text { de los Transportes }\end{array}$ & TRA \\
\hline D. Ingeniería Electrónica & ELN & D. Ingeniería del Terreno & TRR \\
\hline D. Escultura & ESC & D. Ingeniería Textil y Papelera & TXP \\
\hline D. Física Aplicada & FIS & D. Urbanismo & URB \\
\hline
\end{tabular}

El sistema universitario español considera dos categorías principales de docentes dependiendo de su vinculación laboral con la Universidad: profesorado funcionario y no funcionario. La Ley Orgánica 4/2007, de 12 de abril, por la que se modifica la Ley Orgánica 6/2001, de 21 de diciembre, de Universidades reduce los cuerpos de funcionarios docentes universitarios a Catedráticos de Universidad (CU) y Titulares de Universidad (TU), suprimiendo los cuerpos de Catedráticos de Escuela Universitaria (CEU) y Titulares de Escuela Universitaria (TEU). Además se contempla la integración de los cuerpos a extinguir al cuerpo de TU, en el caso de los CEU, automáticamente, previa solicitud, y en el de los TEU, tras su acreditación por la Agencia Nacional de Evaluación de la Calidad y Acreditación (ANECA). De este modo, estas dos categorías han ido paulatinamente desapareciendo (Santos et al.).

Por otra parte, las universidades pueden contratar personal docente e investigador en régimen laboral (no funcionarios). Las modalidades específicas de contratación laboral del ámbito universitario, según el artículo 48, son las correspondientes a las figuras de Ayudante (AYU), Profesor Ayudante doctor (AYUDR), Profesor Contratado doctor (CONDR), Profesor Asociado (ASO) y Profesor visitante (figura no incluida en este estudio puesto que en la fecha de recogida de datos sólo había 1 profesor visitante en toda la UPV), desapareciendo la figura de Profesor Colaborador (COL) contemplada en la LOU $6 / 2001$. Las condiciones y plazos para la convocatoria de plazas de COL a partir de la entrada en vigor de la LO 4/2007 se regularon con el RD 989/2008 de 13 de junio, extendiéndose hasta el 3 de mayo de 2013, de ahí que todavía haya muchos docentes de esta categoría en la UPV. Las categorías del profesorado se 
recogen en la tabla 3, ordenadas de menor a mayor rango y con su equivalente internacional (Sánchez de Madariaga, 2014)

Tabla 3. Categoría del profesorado de la UPV

\begin{tabular}{|l|c|c|}
\hline Asociado & ASO & Grade D \\
Ayudante & AYU & Non PhD Teaching staff \\
Colaborador & COL & Grade C \\
\hline Ayudante doctor & AYUDR & \\
Contratado Doctor & CONDR & Grade B \\
\hline Titular de Escuela Universitaria & TEU & \\
Titular de Universidad & TU & Grade A \\
Catedrático de Escuela Universitaria & CEU & CU \\
\hline Catedrático de Universidad & EME & \\
Emérito & & \\
\hline
\end{tabular}

La Ley de Reforma Universitaria de 1983 estableció la estructura departamental en la Universidad española. El profesorado depende de un departamento y, de éste, parten las decisiones de contratación, siguiendo las directrices generales de la Universidad (Escolano, 2006, 125). Por ello, resulta interesante analizar la situación del profesorado adscrito a los distintos departamentos desde la perspectiva de género.

Se han utilizado las siglas $\mathrm{H}$ para hombres y $\mathrm{M}$ para mujeres además de los símbolos î respectivamente. En los gráficos de barras, su altura representa el número de docentes en valor absoluto, completando este dato con el porcentaje que representan. Los valores de los porcentajes, calculados con decimales, se han redondeado para ajustarlos al $100 \%$.

\section{RESULTADOS Y DISCUSIÓN}

\subsection{Representación de las mujeres en el PDI de la UPV}

Si bien según las estadísticas del Ministerio de Educación Ciencia y Deporte (MECyD 2015a) el porcentaje de mujeres matriculadas en los centros propios de la UPV durante el curso 2014-2015 en estudios de grado, primer y segundo ciclo fue del $35 \%$, el análisis de la totalidad del profesorado de la UPV muestra que sólo el 30\% del PDI son mujeres (Figura la). Este porcentaje es inferior al $39 \%$, correspondiente a la media del PDI de las universidades públicas españolas en 2012 (MEC-UMYC) o al 39,4\%, media nacional en las universidades públicas el curso 2014-15 (MECyD 2015b). Esto podría deberse a que, como se ha mencionado en los antecedentes, la rama de conocimiento Ingeniería y Arquitectura (históricamente masculinizada) es la más representativa, 
englobando al 66\% de los grados ofrecidos. De hecho, en la mayoría de países de la UE la mayor desigualdad de género entre el PDI se da en la Ingeniería, y la menor en las Humanidades (Pastor y Acosta, 2016)

Aunque es superior al porcentaje femenino de las Universidades Politécnicas de Madrid y Barcelona (24\%) es de señalar que apenas ha aumentado un punto desde el año 2012 (Marqués et al.). Así, el cambio está siendo muy lento, a pesar de que a nivel nacional dentro del PDI de Ingeniería y Arquitectura las mujeres tienen más sexenios que sus colegas varones (68,1\% de las funcionarias los tienen, frente al 65,4\% de los hombres). Ingeniería y Arquitectura es, de hecho, la única rama del conocimiento en que ellas les superan (Guil, 2016). Resulta difícil de entender que a día de hoy, pese a estar más preparadas, las mujeres no ocupen al menos las mismas posiciones que los hombres, siendo la única justificación posible que, habiendo copado los hombres dichos puestos en el pasado, y dada la baja tasa de reposición consecuencia de las recientes políticas de austeridad, no salgan plazas a concurso a las que optar.

Según la Figura 1b, más de la mitad del profesorado (59\%) pertenece a los cuerpos de funcionarios docentes. Sin embargo, apenas el 16\% del PDI son profesoras funcionarias, frente al $43 \%$ de los hombres.
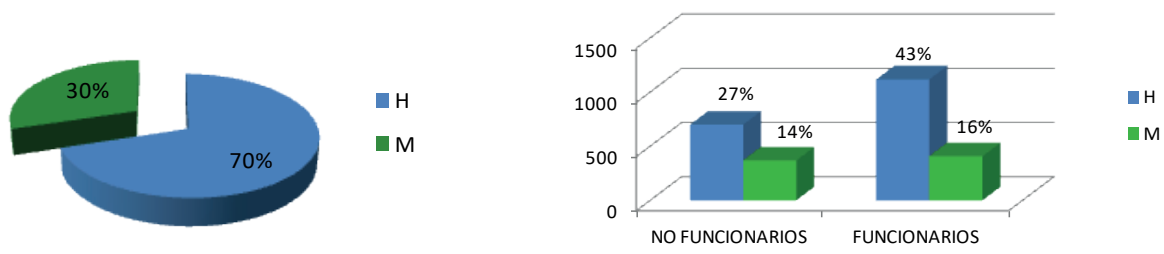

Figura 1a. Distribución del PDI en UPV

Figura 1b. PDI funcionario/contratado en UPV

La Figura 2 refleja la distribución por género de las distintas categorías docentes en la UPV. Una distribución desigual es un indicador de segregación vertical, es decir, de jerarquía laboral, de desigualdad salarial y de desarrollo de mayores niveles de poder en el contexto universitario de un sexo respecto a otro; en este caso, de los hombres respecto a las mujeres.
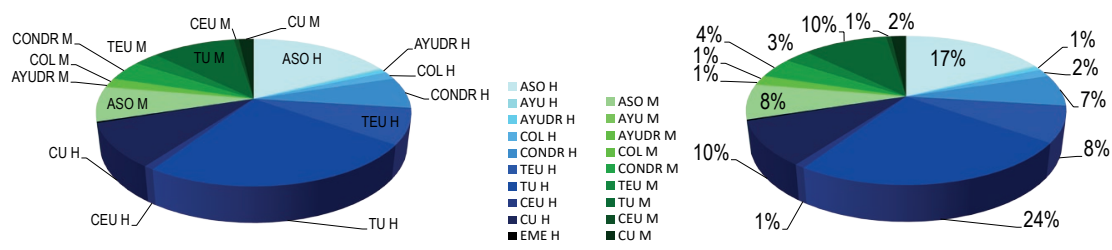

Figura 2 Distribución por género de las categorías docentes en la UPV 
Analizando los datos del PDI por categorías, se puede resaltar el reducidísimo porcentaje de académicas que se encuentran en la cima de la pirámide jerárquica: sólo el 2\% son CU, (equivalente a Grado A), frente al 10\% de los hombres. En cuanto a las categorías correspondientes a Grado B (TEU, TU y CEU), el 16\% son mujeres y el 33\% hombres, y en las categorías del Grado C (no funcionarios), el 5\% de mujeres frente al 8\% de hombres.

En la Figura 3 se comparan, por categorías, el número y porcentaje de profesorado femenino y masculino en la UPV. El diagrama de líneas evidencia las diferencias entre las distintas categorías, siendo la brecha mayor en el cuerpo de CU. No existe en la UPV ninguna categoría profesional que esté feminizada, aunque en las categorías inferiores de AYUDR y COL los porcentajes entre sexos se aproximan algo más. El principal motivo podría ser que estas figuras profesionales son relativamente recientes y, dada su precariedad, las mujeres podían optar a ellas en el momento de su creación. Sin embargo, la llegada de la crisis económica hizo que también fueran demandadas por los hombres alcanzando una cierta paridad.

Es indiscutible que en las figuras más valoradas por su estabilidad y por el conjunto de derechos profesionales que comportan, los hombres siguen siendo una aplastante mayoría, que se incrementa todavía más en el vértice de la pirámide, donde la presencia femenina resulta meramente simbólica.
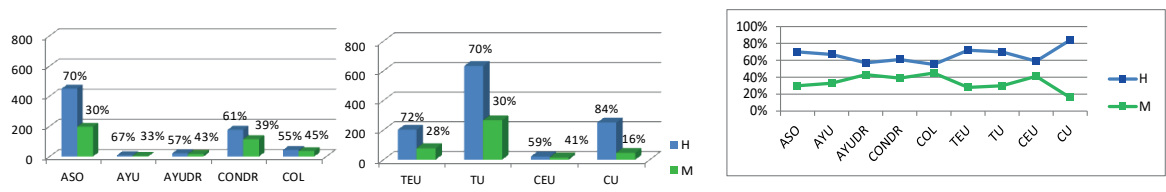

Figura 3. Distribución del profesorado UPV por categorías (no funcionarios/funcionarios)

Según los datos del Instituto Nacional de Estadística de 2006 (INE), nueve años antes había en la UPV un total de 1715 docentes funcionarios, de los cuales 1299 eran hombres y 416 mujeres. En 2015 hay 1520 docentes funcionarios, 1112 hombres y 408 mujeres, produciéndose, por tanto, una reducción total del $11,3 \%$ de profesorado funcionario. Aunque las gráficas de barras de la Figura 4 parecen mostrar que la situación de las mujeres ha mejorado, la gráfica de líneas ilustra bien que la brecha sólo se ha reducido en el colectivo de CEU, categoría a extinguir. Sin embargo, en el resto de las categorías, en las que apenas se ha modificado la brecha, todavía estamos muy lejos de la igualdad.

Los porcentajes obtenidos para la UPV se han comparado con los datos del curso 2013-2014 del resto de las universidades públicas españolas proporcionados por el Ministerio de Educación Ciencia y Deporte (MECyD, 2015b) y 
por el Ministerio de Economía y Competitividad (MEC-UMYC, 2014) y con los datos de las universidades europeas proporcionados por la publicación «She figures 2015» (European Comission, 2016).
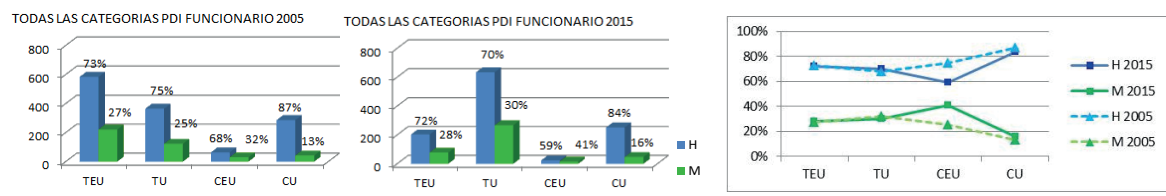

Figura 4. Comparación del profesorado funcionario de la UPV en 2005 y 2015

El denominado «efecto tijera» que hace referencia al hecho de que conforme se va ascendiendo en las responsabilidades y cargos, como cátedras, por ejemplo, aumenta el número de hombres y desciende el de mujeres, queda bien representado en la Figura 5. Asimismo muestra que en el caso de la UPV las hojas de las tijeras están más alejadas que en el resto de España y que en Europa: el 84\% de las cátedras (Grado A) de la UPV está en manos de hombres, y solo el $16 \%$ en manos de mujeres, mientras que en el resto de universidades españolas esta última cifra es 20,7\% y en las europeas $20.9 \%$. El 33\% del PDI de la categoría Grado B (CEU+TU+TEU), son mujeres, de nuevo porcentaje inferior al del resto de universidades españolas $(36,7 \%)$ y europeas $(37,1 \%)$. En cuanto a la categoría C, el $41 \%$ son mujeres, frente al $48,5 \%$ en el caso español y el $45,1 \%$ en el europeo.
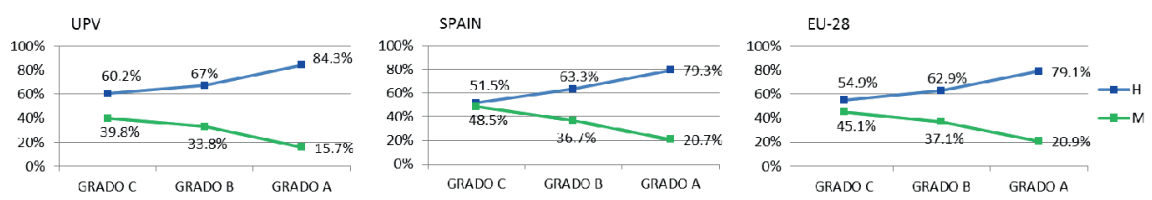

Figura 5. Distribución del profesorado por categorías: UPV, España y Europa-28

\subsection{Representación de las mujeres PDI en los centros docentes de la UPV}

Estudiando las vías de inserción de las profesoras en las distintas disciplinas, puede determinarse si se han superado o se están superando los modelos tradicionales de presencia femenina en el mundo académico. Esto es, si ellas siguen ligadas a carreras extensivas de los roles y tareas domésticas o, utilizando la denominación más común, vinculadas a carreras «de letras», alejadas de las carreras más científicas y técnicas (De los Cobos; Escolano 2006; Lecuona). 
La asignación de la docencia del profesorado de la UPV en sus centros docentes (9 Escuelas, 2 facultades y 2 institutos politécnicos) se representa en la Figura 6, con los centros ordenados de mayor a menor presencia femenina (Figura 6b). Únicamente en la ETSIAMN, en FADE y FBBAA el porcentaje de mujeres PDI supera el $40 \%$. Hay que destacar que ninguna de estas tres instituciones pertenece a la rama de conocimiento de Ingeniería y Arquitectura.
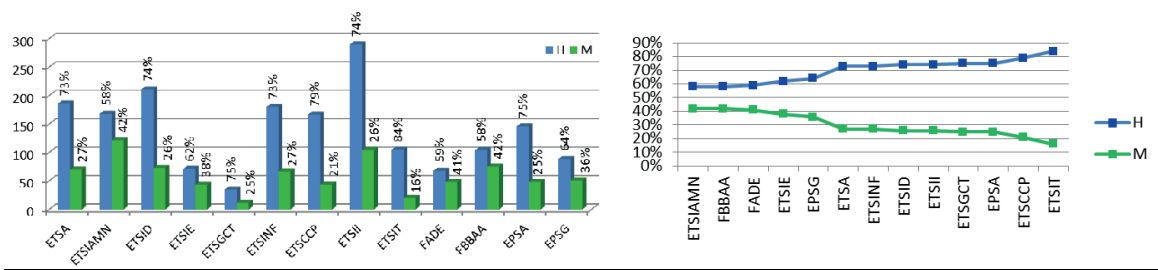

Figura 6 a. Distribución del PDI/centros

Figura 6 b. PDI/centros (\% decreciente de M)

La ETSIAMN pertenece a la rama de Ciencias, y oferta, entre otros, los grados de Ingeniería agroalimentaria y del medio rural, y Ciencia y tecnología de los alimentos. Estos estudios tienen relación con los alimentos, la comida, la nutrición, la restauración y la industria alimentaria en general, áreas tradicionalmente más afines a la mujer y ligadas a los estereotipos de género. Además, los estudios de Ciencia y tecnología de los alimentos cumplen su 20 aniversario en 2017, con lo cual son relativamente recientes y han podido incorporar a más mujeres a medida que han crecido. En cuanto a FADE, está vinculada a Ciencias Sociales y Jurídicas, y FBBAA a Artes y Humanidades, áreas en las que la mujer ha estado siempre más representada (Lecuona).

En el polo opuesto se encuentran la ETSCCP y la de ETSIT, que son las más masculinizadas, con una participación escasa de la mujer (21\% y 16\% respectivamente). Ambas escuelas pertenecen a la rama de conocimiento Ingeniería y Arquitectura. Además, la E.T.S. de Caminos Canales y Puertos mantiene estrecha relación con la construcción, un sector caracterizado por la existencia de numerosas barreras al desarrollo profesional de las mujeres (Navarro-Astor et al.).

Si se analizan estos datos, teniendo en cuenta las distintas categorías entre profesorado funcionario por un lado y no funcionario por otro, se observa (Figura 7) que la brecha es menor entre el profesorado no funcionario, invirtiéndose incluso los valores en la ETSIAMN (53\% de mujeres). Por el contrario, la desigualdad es mayor entre el profesorado funcionario. 

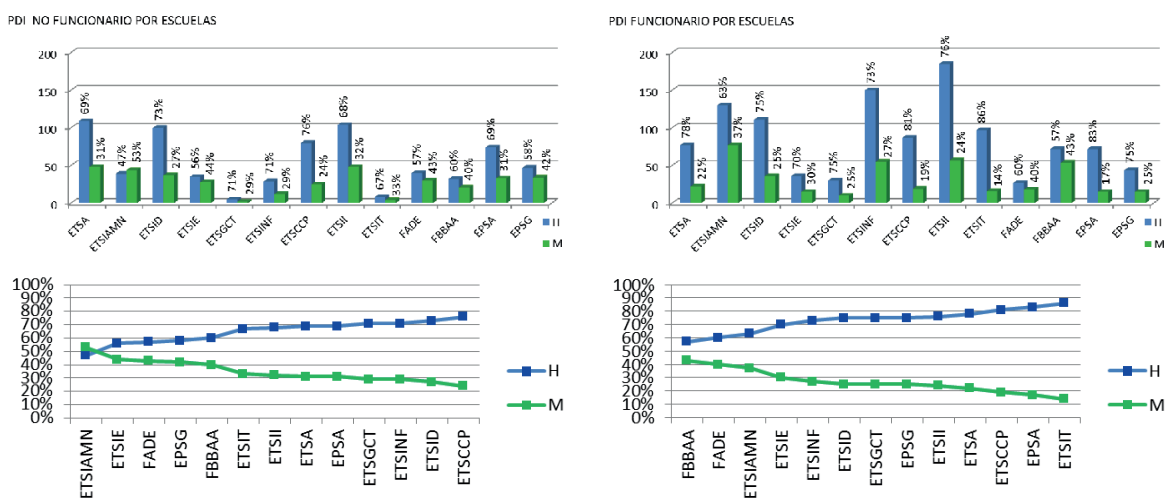

Figura 7. Distribución del PDI no funcionario y funcionario en cada centro docente

Las gráficas de las Figuras $8 \mathrm{a}$ y $8 \mathrm{~b}$ muestran el porcentaje de hombres y mujeres del PDI adscrito a cada centro desagregado por categorías. Los diagramas de barras reflejan los porcentajes de hombres y mujeres de cada categoría y las figuras de la derecha el porcentaje respecto a la totalidad del profesorado adscrito al centro.

Las desigualdades por categorías en cada centro quedan reflejadas en la Figura 9. En la ETSIT, ETSID, ETSINF y EPSG la línea que representa a las mujeres no se cruza en ninguna categoría con la de los hombres, mientras que en la ETSCCP, EPSA y FBBAA hay alguna categoría en la que coinciden ambas (categorías del grado C y D en ETSCCP, EPSA y TU en FBBAA). En la ETSA, ETSIE, ETSIAM y FADE se producen cruces entre las líneas en las categorías de rango inferior (COL, CONDR, AYUDR). Aunque en la ETSII y ETSIGCT las CEU superan a los CEU, no hay que olvidar que es una categoría a extinguir. Finalmente, en FADE, facultad de reciente creación (2002) perteneciente al área de conocimiento de Ciencias Sociales, se iguala el porcentaje de TU, CEU y presenta menor brecha en $\mathrm{CU}$. 


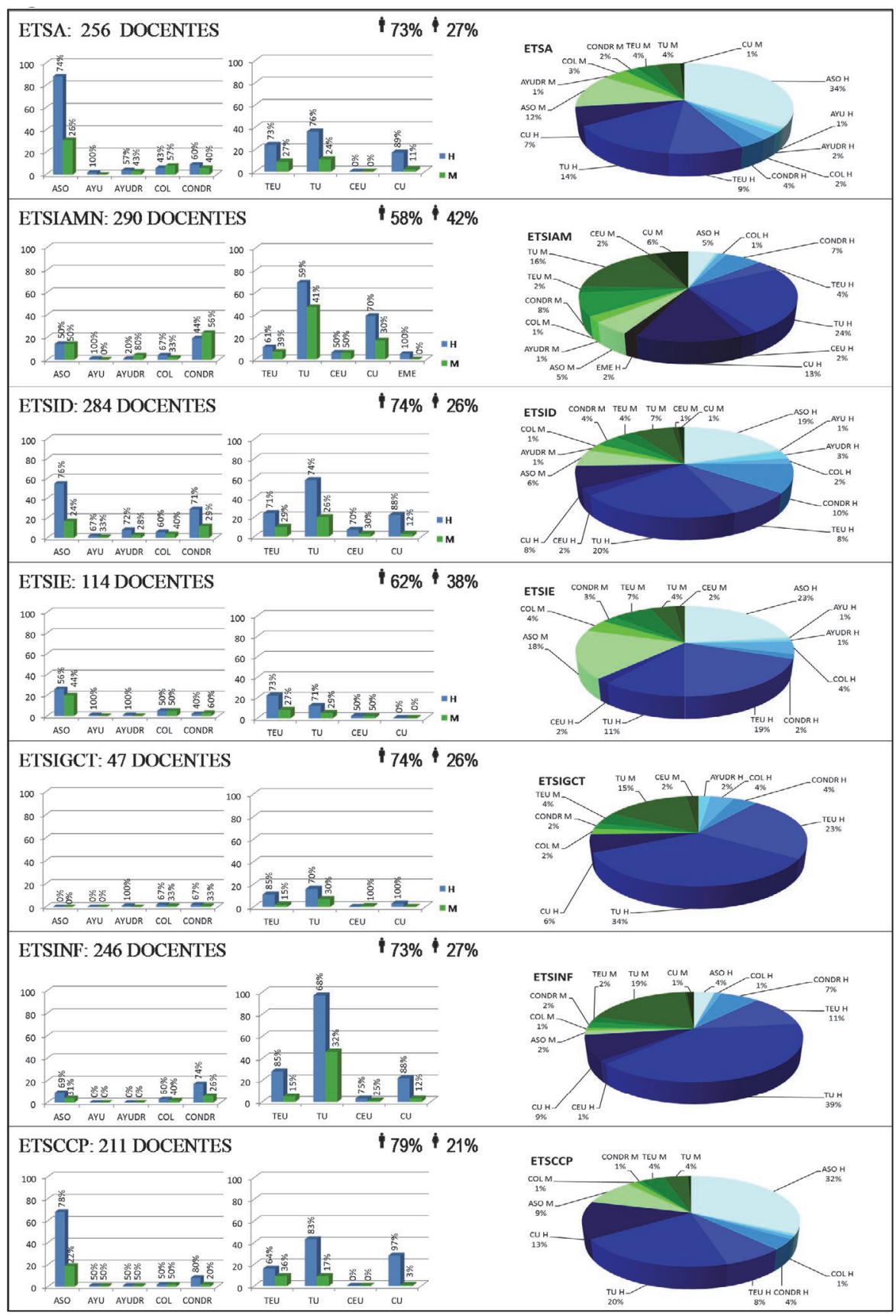

Figura 8a. Distribución del profesorado por categorías (no funcionarios/funcionarios) y centro 


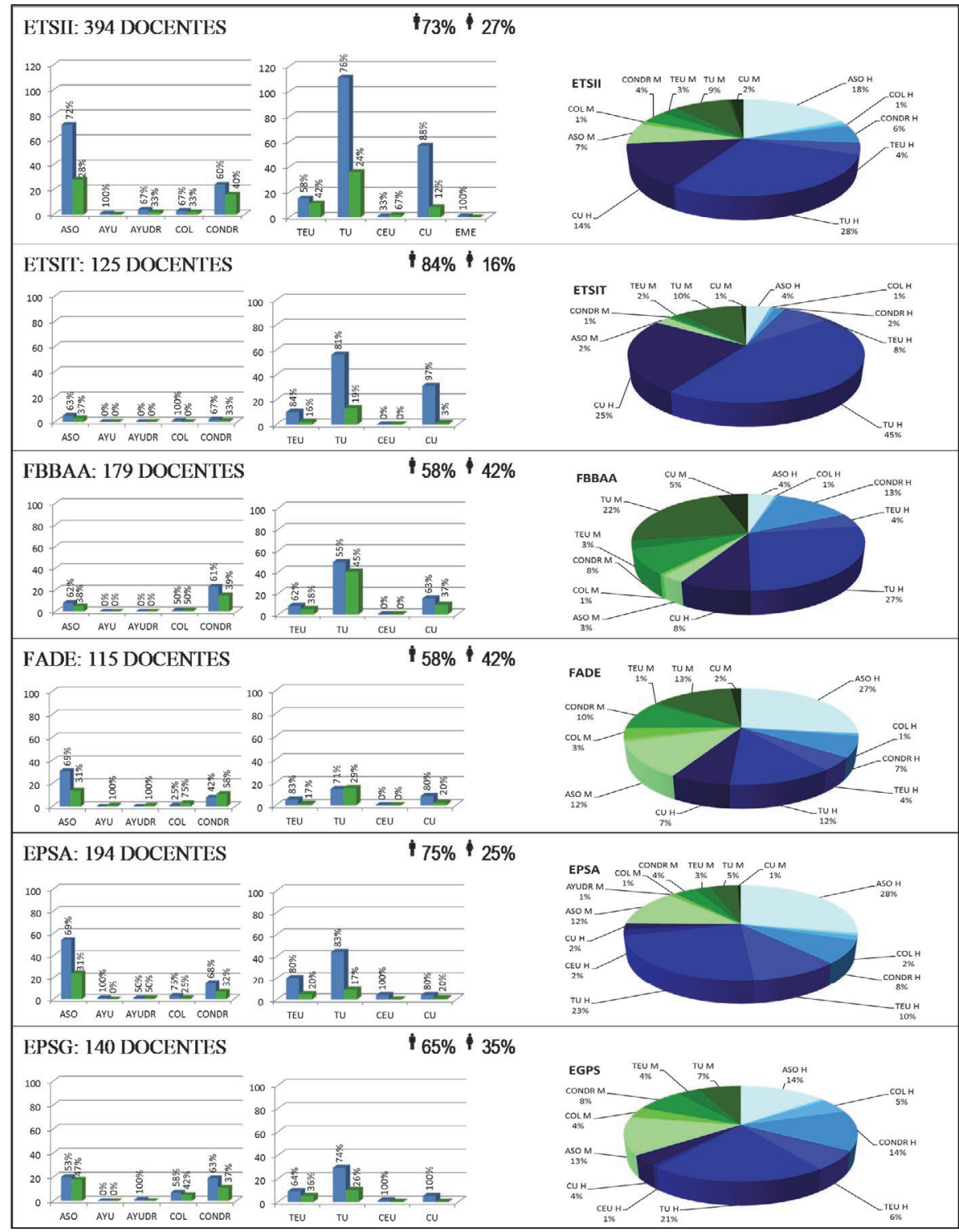

Figura 8b. Distribución del profesorado por categorías (no funcionarios/funcionarios) y centro 

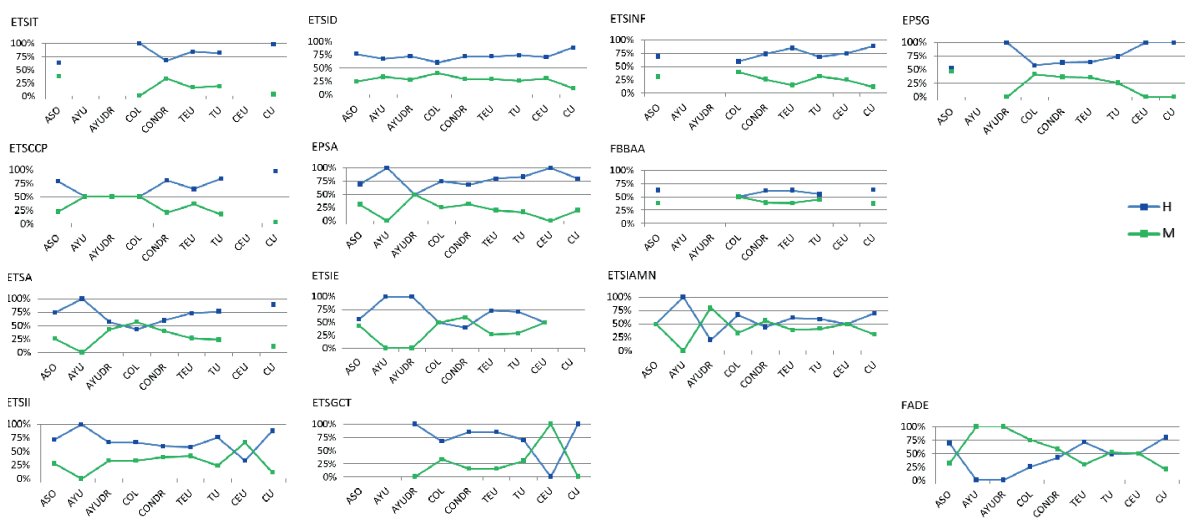

Figura 9. Desigualdades por categorías en cada centro

\subsection{Representación de las mujeres PDI en los departamentos de la UPV}

Dado que son los departamentos los que contratan al personal no funcionario y los que convocan las correspondientes plazas para las figuras de profesor funcionario, tal y como se ha comentado anteriormente, se ha creído conveniente analizar la distribución por sexos del profesorado de los 42 departamentos de la institución objeto de estudio.

Los resultados de este análisis (ver Figura 10) reflejan una gran disparidad entre departamentos: IDM es el más feminizado con mayor porcentaje de profesoras (83\%), mientras que ISA es el más masculinizado, con tan sólo un 4\%.

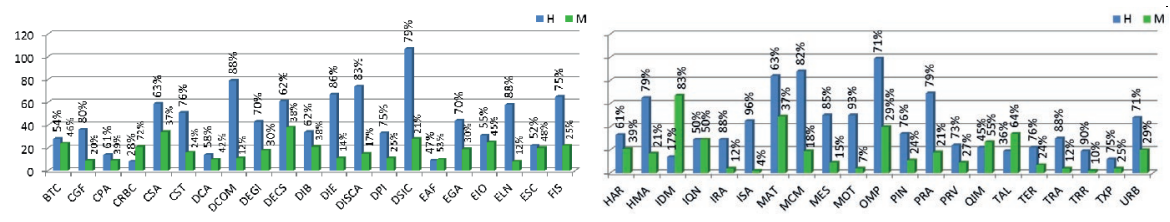

Figura 10. Distribución del PDI por departamentos

Ordenando los departamentos según su participación femenina (Figura 11) se observa que sólo 3 departamentos tienen una cuota femenina de PDI claramente superior a la masculina (IDM, CRBC y TAL). Hay que subrayar que en 2001 los dos primeros ya tenían mayoría femenina y que TAL empleaba un $48.6 \%$ de mujeres (Escolano 2006), y que ninguno pertenece al campo científico de Ingeniería y Arquitectura. 
Tecnología de los Alimentos (TAL) se vincula a Ciencias en general, y al ámbito de la cocina (típicamente femenino). Conservación y Restauración de los Bienes Culturales (CRBC) pertenece a Artes y Humanidades y tiene que ver con el cuidado y mantenimiento de bienes importantes para la sociedad. Finalmente, Idiomas (IDM), del campo de las Ciencias Sociales, ha estado siempre vinculado con la enseñanza, ámbito eminentemente femenino.

La Figura 11 también muestra que 6 departamentos (QIM, EAF, IQN, ESC, BTC, EIO) tienen un reparto bastante igualitario cercano al 50\%. Los 7 departamentos siguientes tienen entre un 40-50\% de mujeres, desde EGA hasta PRA tienen entre el 20 y $30 \%$, mientras que los 12 departamentos restantes tienen menos del $20 \%$ de profesoras.

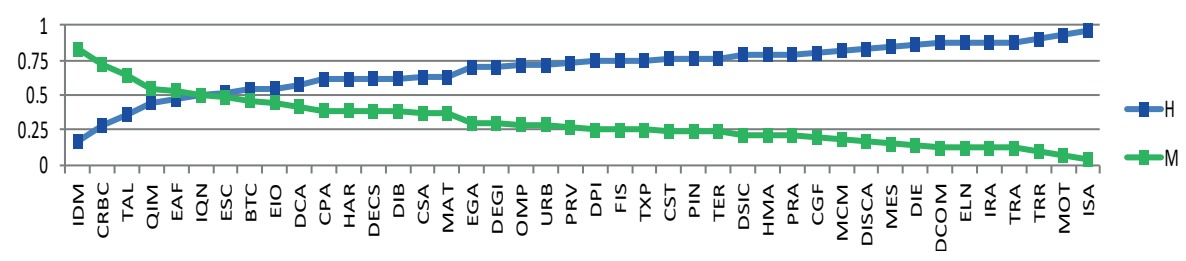

Figura 11. Distribución del PDI por departamentos (orden decreciente de mujeres)

Si este análisis se lleva a cabo distinguiendo entre docentes funcionarios y no funcionarios (ver Figura 12), llama la atención la existencia de dos departamentos que carecen de profesoras funcionarias (TRA y PRA). Además conviene subrayar que el primero, TRA, es también uno de los tres departamentos que carecía de profesoras en 2001 (Escolano, 2006), denotando la ausencia de evolución hacia la igualdad en catorce años.

En cuanto al segundo, PRA, está compuesto exclusivamente por arquitectos, profesión cuyos estudios de grado están feminizados hace muchos años (Basset-Salom et al.; Sánchez de Madariaga 2014). Así, el simple paso del tiempo no ha contribuido a mejorar las cifras de igualdad. Este departamento está siendo objeto de estudio exhaustivo de otra investigación, cuyos resultados preliminares señalan que a igualdad de fechas de graduación y de acceso a la carrera profesional y docente en la figura de profesor asociado, la maternidad ha supuesto una traba en la promoción profesional de las mujeres que no se percibe en los hombres.

Asimismo, se observa que los departamentos con mayor número de funcionarios (DCOM, DIE, DISCA, DSIC, ELN, FIS) presentan porcentajes de profesoras inferiores al 20\%, a excepción de MAT, en el que la cuota femenina alcanza el 35\%. 


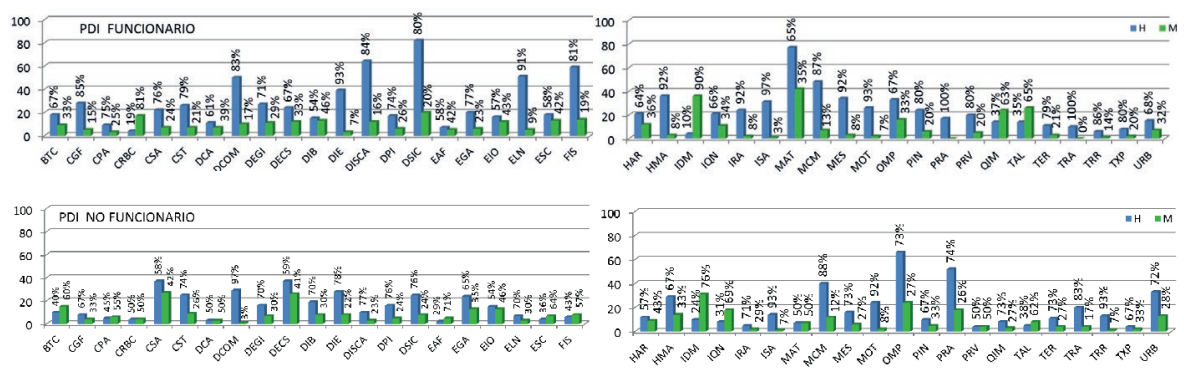

Figura 12. Distribución del PDI funcionario/no funcionario por departamentos

En la Figura 13 se observa que la brecha en el profesorado no funcionario es menos acusada, salvo las singularidades de DIB, DPI, OMP, MCM, DCOM, TRR, MOT, y ISA, además de llamar la atención la inversión en los departamentos de QIM y DPI.

PDI FUNCIONARIO

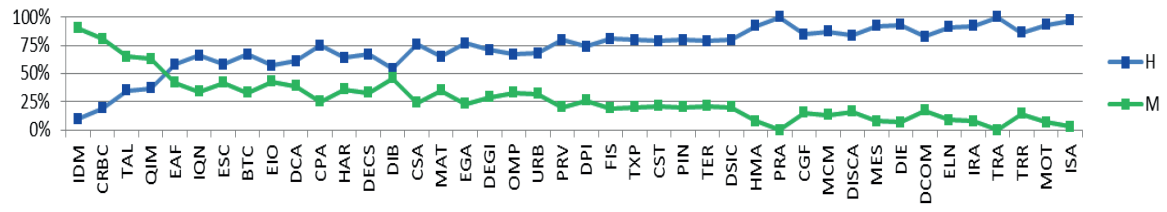

PDI NO FUNCIONARIO

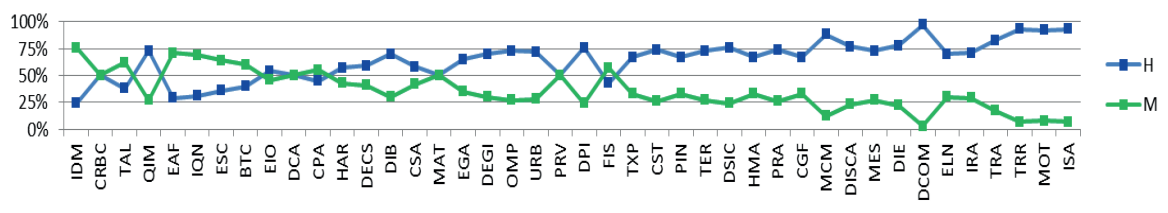

Figura 13. Desigualdades en la distribución del PDI por departamentos

Este análisis se completa calculando los porcentajes para cada una de las categorías de docentes en cada departamento. Los resultados se muestran en las Figuras 14a, 14b, 14c, 14d, 14e y 14 f. 
La Figura 15 evidencia claramente las desigualdades. En un 29\% de los departamentos (enmarcados en rojo) la proporción de hombres es mayor para todas las categorías (aquéllos en los que las líneas no se cortan): CGF, CST, DCOM, DIE, DISCA, DSIC, HMA, ISA, URB, TER, TRR, y MOT, y su profesorado supone el $42 \%$ del PDI de la UPV.

Sólo en 3 departamentos (enmarcados en verde) TAL, CRBC e IDM, el porcentaje de mujeres es superior en todas las categorías. En los restantes predominan en general los hombres especialmente en las figuras de mayor categoría, abriéndose las puntas de las tijeras conforme se acercan a la figura de CU.

Esta descripción de la distribución del profesorado por departamentos es imprescindible y fundamental para reflexionar acerca de las posibles causas de la desigualdad. No hay que olvidar que el director/a de cada departamento es quien solicita a Rectorado la creación de nuevas plazas docentes, y quien nombra las comisiones que han de juzgar los méritos de los aspirantes a las distintas figuras laborales, así como los tribunales para las plazas de funcionario. Además, dichas comisiones están formadas por tres miembros elegidos por el Rector, y dos designados por el Consejo del Departamento al que se adscribe la plaza, todos pertenecientes a cuerpos de funcionarios docentes universitarios y con titulación oficial igual o superior a la de la plaza convocada (colectivos masculinizados, como se aprecia en la Figura 15). Considerando que el 85\% de los directores de los departamentos de la UPV son hombres (Guardiola-Víllora et al.), y habiendo detectado tras un análisis no exhaustivo de la composición de las últimas comisiones de evaluación nombradas en 2016-17, que la mayoría apenas tiene un $20 \%$ de representación femenina, se podría pensar que el colectivo masculino favorece a sus colegas. 
El profesorado de la Universitat Politècnica de València desde la perspectiva de género 265

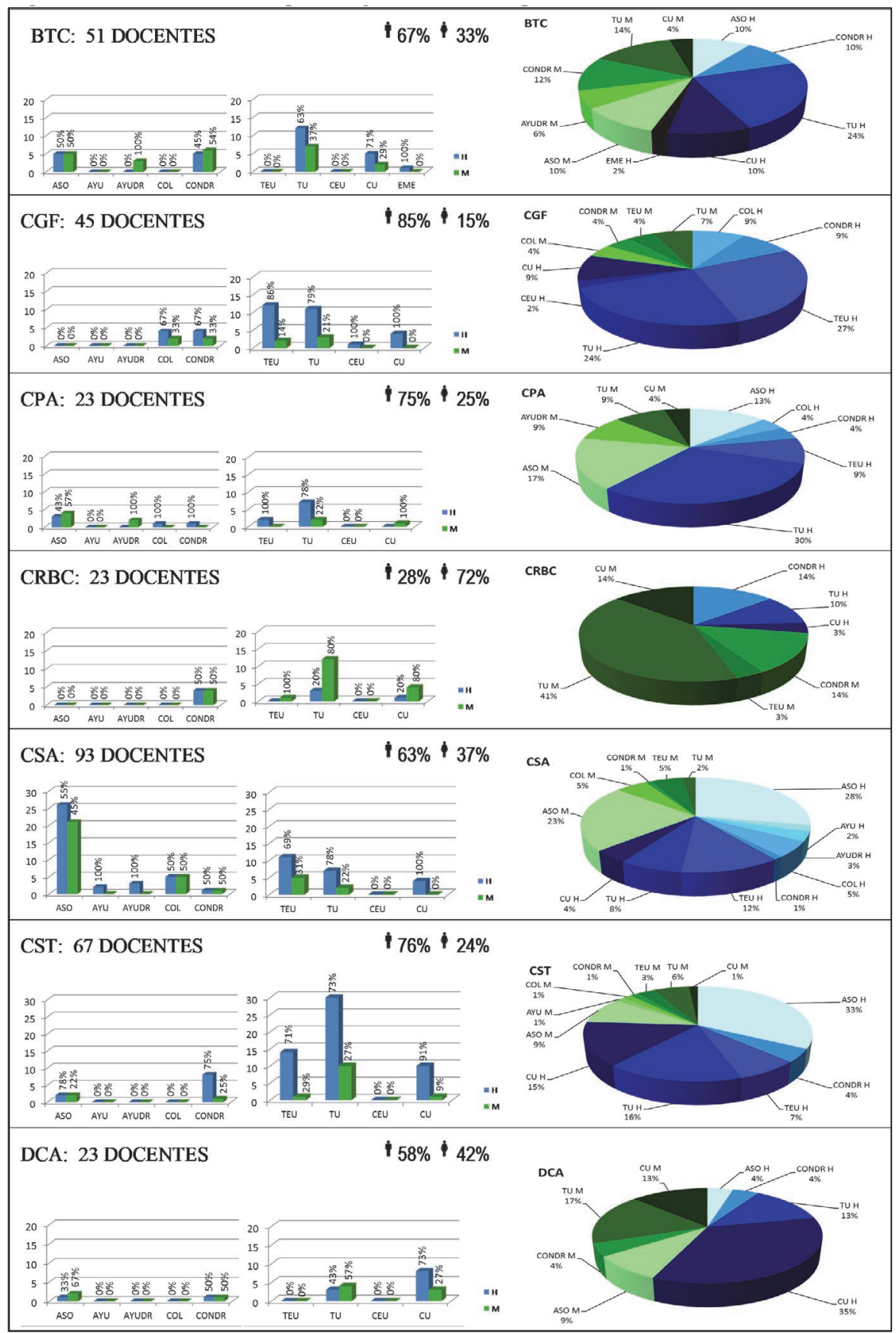

Figura 14a. Distribución PDI por categorías en cada departamento

Feminismo/s 29, junio 2017, pp. 243-277 


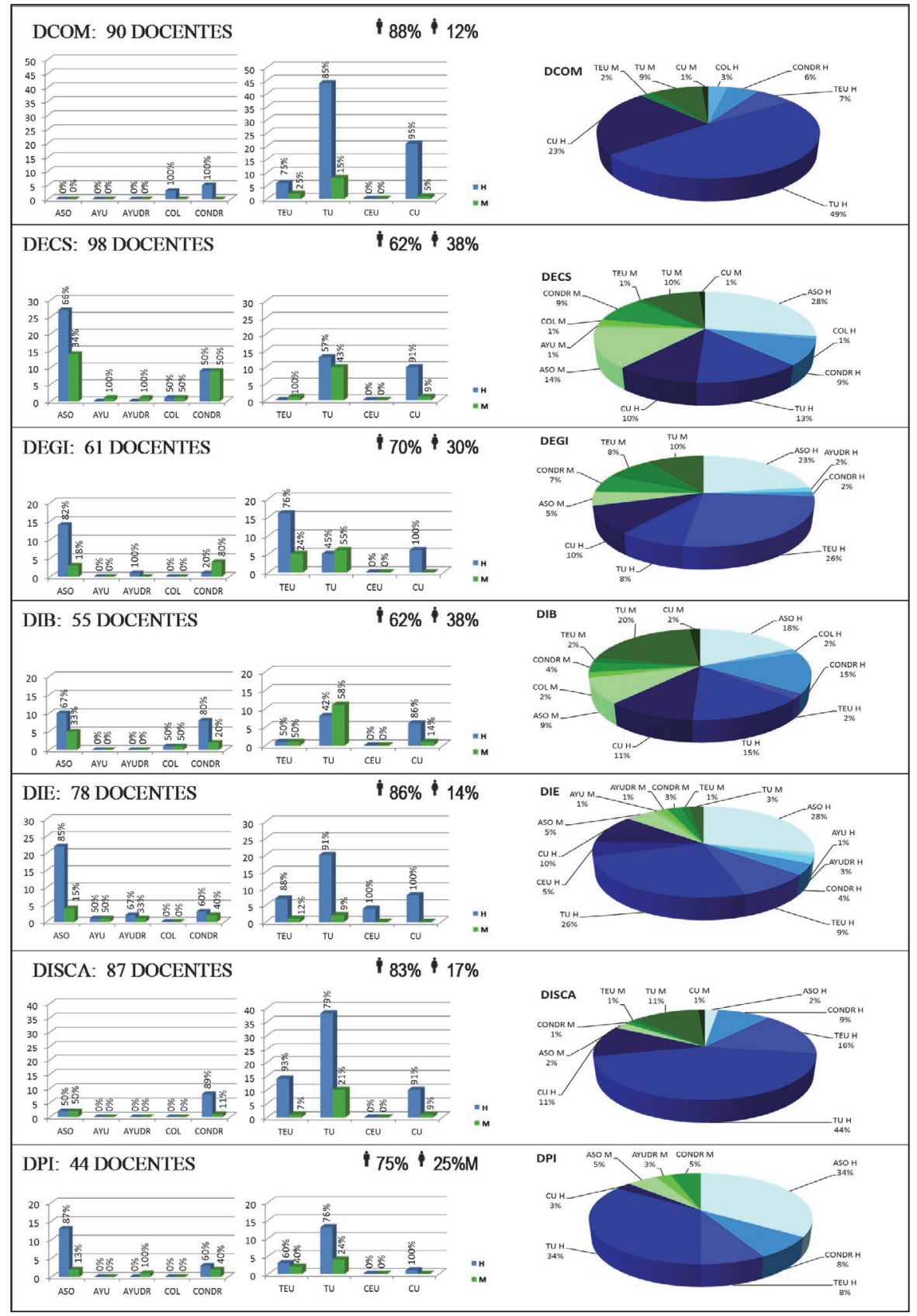

Figura 14b. Distribución PDI por categorías en cada departamento 


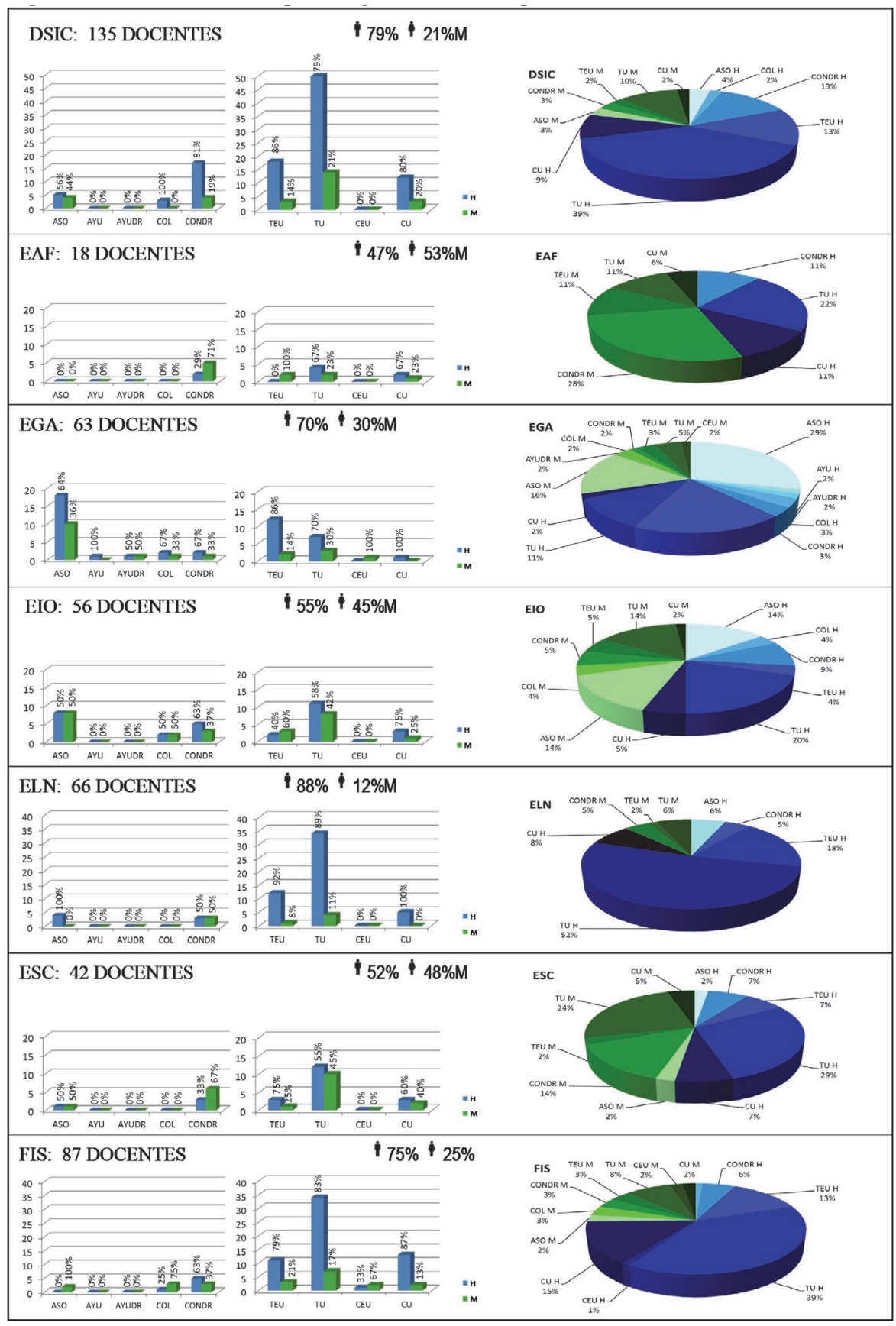

Figura 14c. Distribución PDI por categorías en cada departamento 


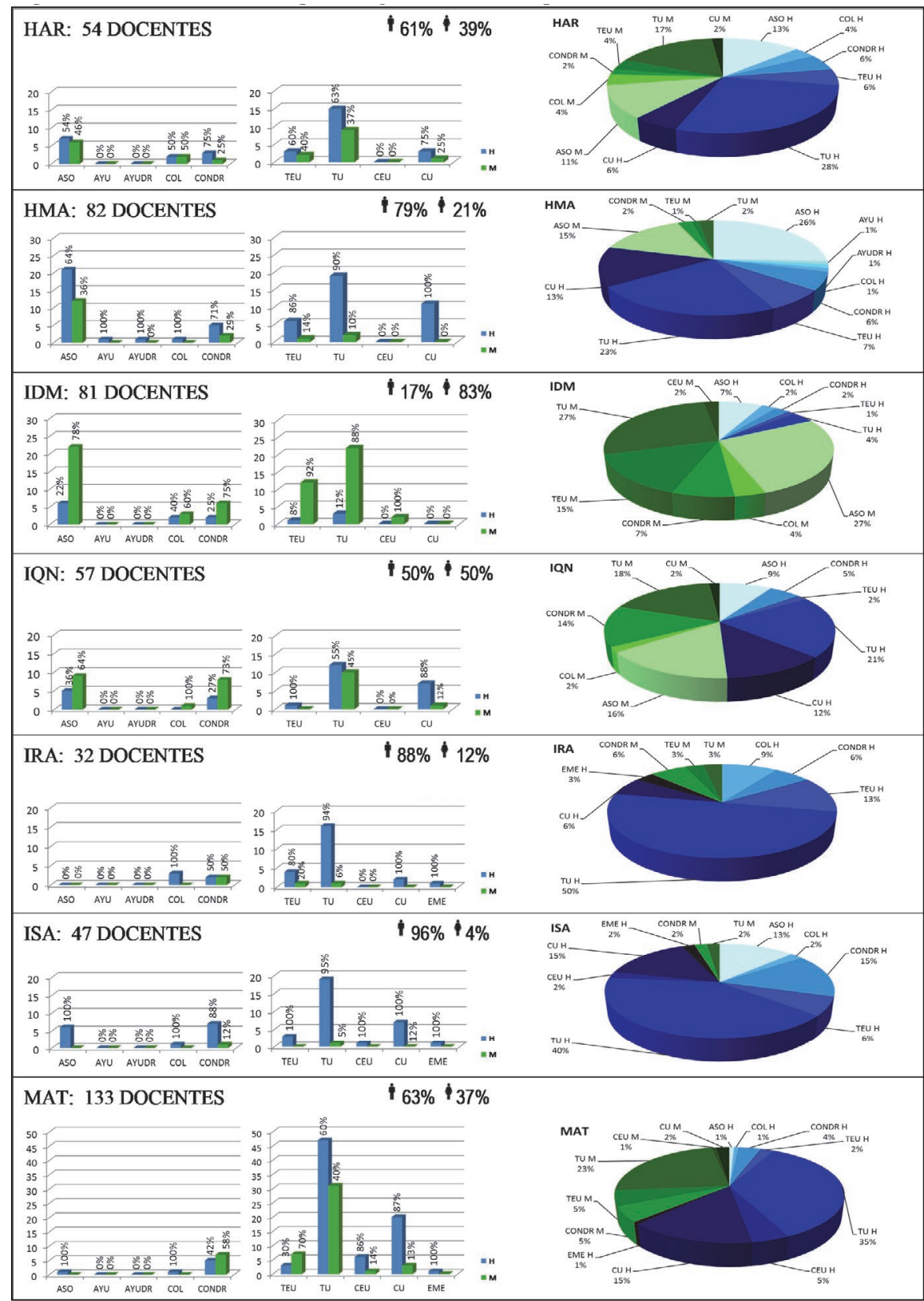

Figura 14d. Distribución PDI por categorías en cada departamento 


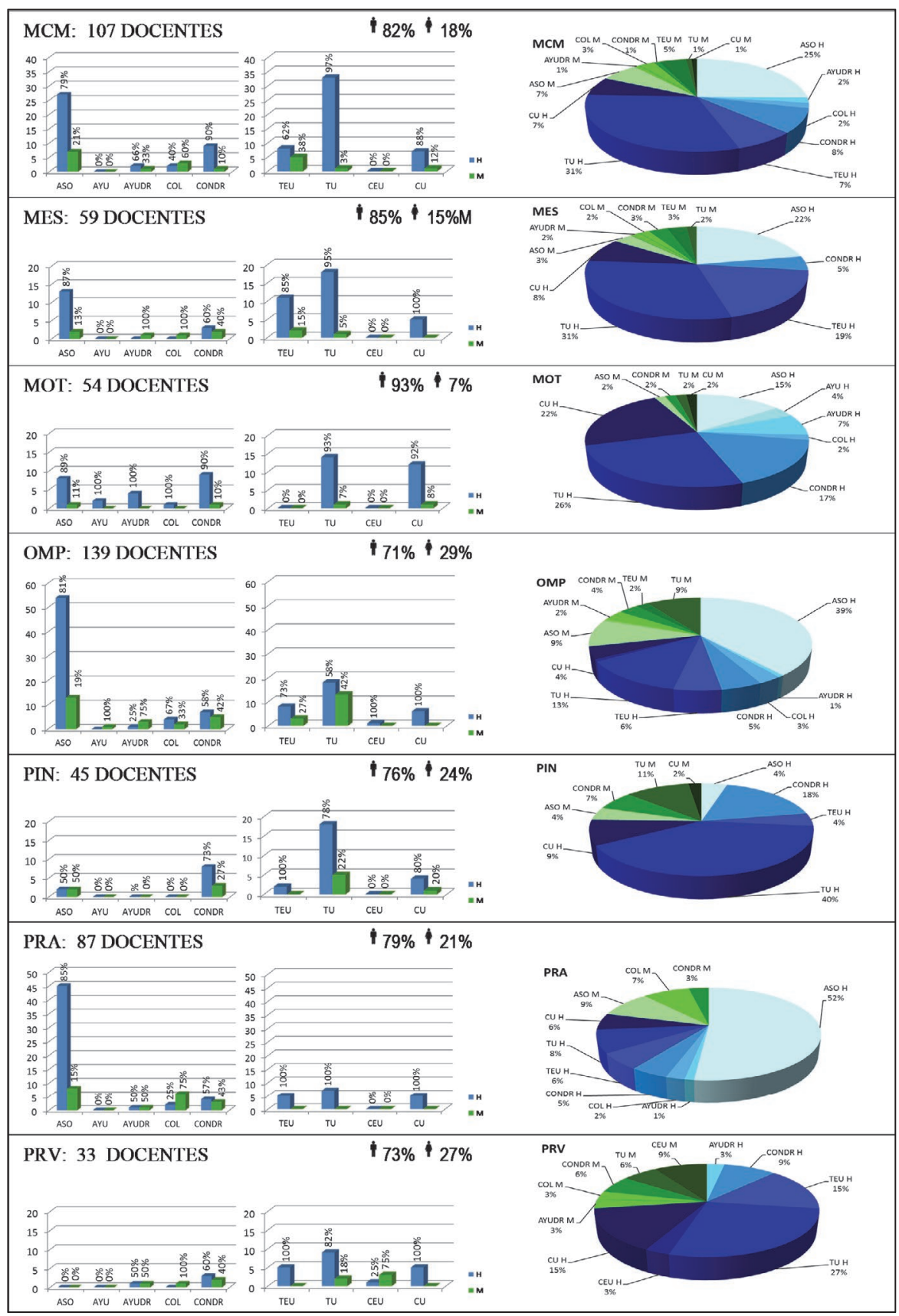

Figura 14e. Distribución PDI por categorías en cada departamento 


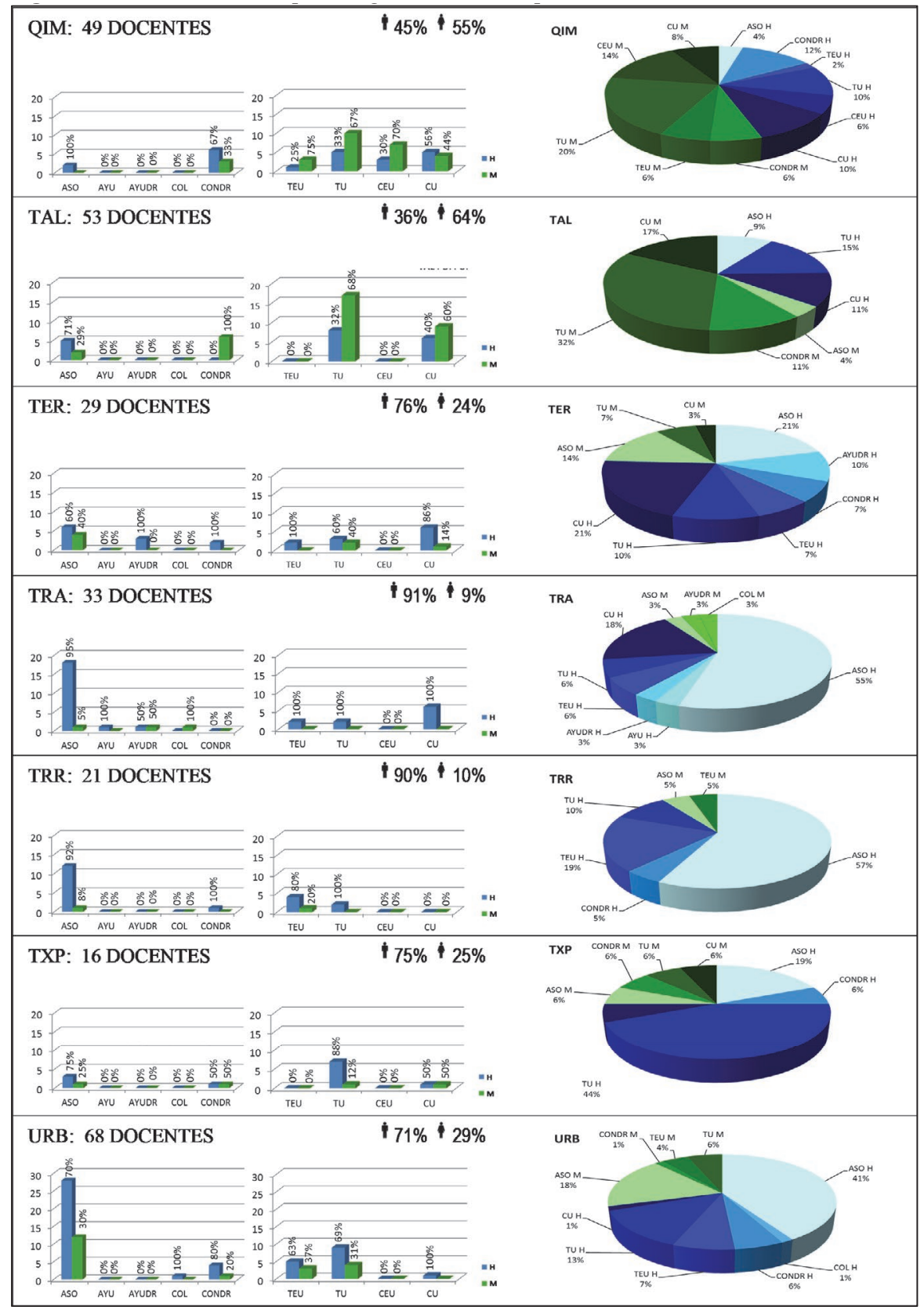

Figura 14f. Distribución PDI por categorías en cada departamento 


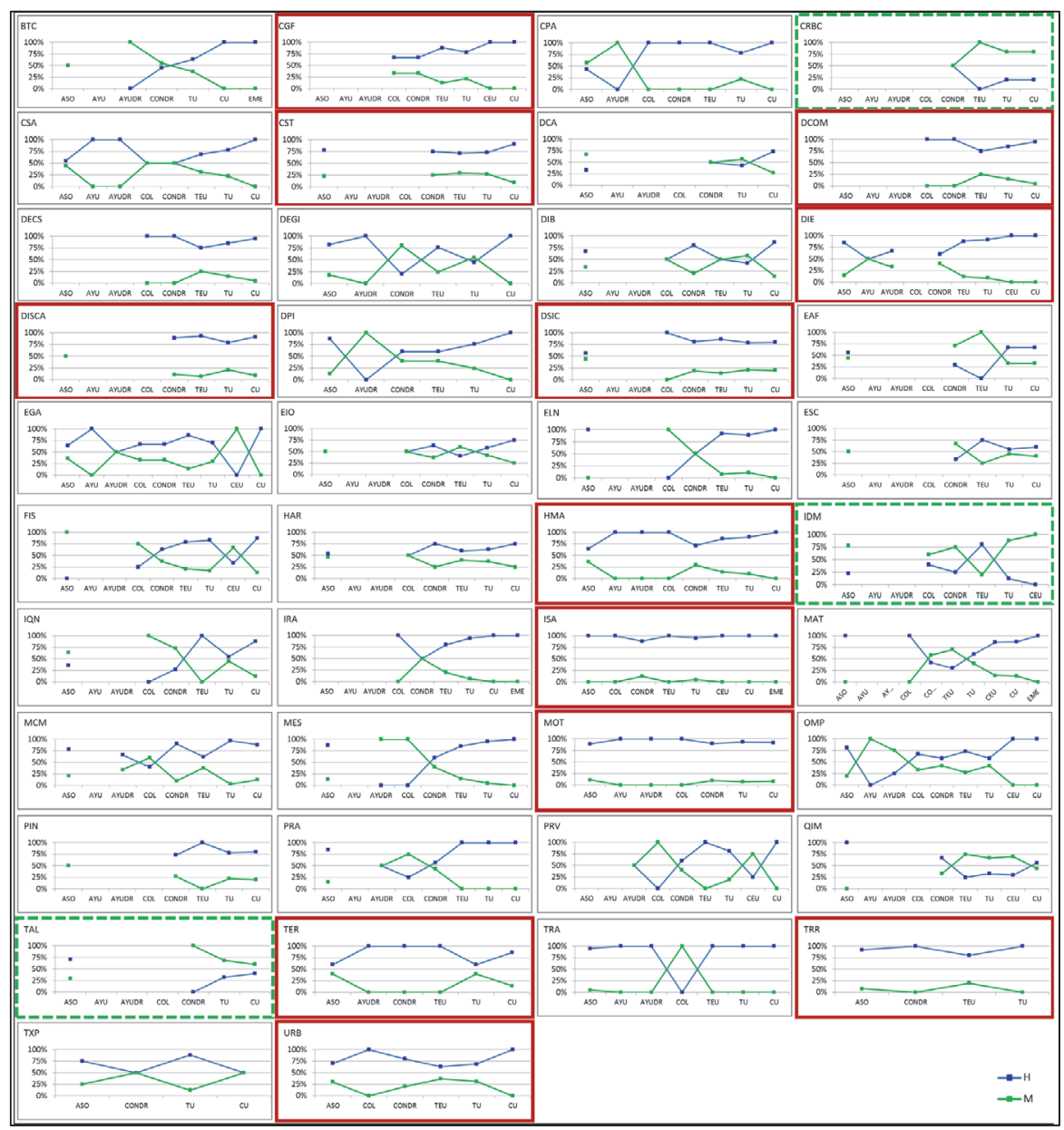

Figura 15. Desigualdades en la distribución PDI por categorías en cada departamento

\section{CONCLUSIONES}

El retrato de la situación del profesorado de la UPV desde la perspectiva de género muestra las siguientes características:

La presencia de las mujeres como docentes está muy alejada de los niveles masculinos, no representando siquiera un tercio (30\%). Esta distribución se aleja de lo que la Ley Orgánica 3/2007 de 22 de marzo, entiende por «composición equilibrada» definida como aquella situación en la que las personas de cada sexo no sean menos del $40 \%$. 
La UPV presenta un importante grado de segregación horizontal: considerando las diversas entidades que la componen (escuelas y facultades), solo tres alcanzan la "composición equilibrada», superando apenas el 40\% (ETSIAMN, FADE y FBBAA), es decir, el 66\% de los centros no alcanza la situación igualitaria. Además, hay que destacar que dos escuelas presentan un porcentaje de profesoras inferior al 25\% (ETSCCP y ETSIT).

En cuanto a los departamentos, se aprecia un ligero avance desde el 2001, en que el $71,5 \%$ de departamentos tenía menos del $25 \%$ de presencia femenina, mientras que en diciembre de 2015, el porcentaje se reduce al $60 \%$ de los departamentos (o un $40 \%$ si se incluye el profesorado no funcionario).

En relación a los departamentos MOT, TRA e IRA, sin mujeres en el 2001, han aumentado ligeramente su presencia, con un $7 \%, 12 \%$ y $12 \%$ respectivamente. Sin embargo, a fecha de hoy, ni el departamento de transportes (TRA) ni el de proyectos arquitectónicos (PRA) cuenta con mujeres entre su profesorado funcionario.

Por otro lado, la UPV presenta sobretodo un importante grado de segregación vertical: a medida que se asciende en la jerarquía académica la proporción de mujeres disminuye. En el análisis del profesorado por categorías, sólo se alcanza el $40 \%$ entre las figuras AYUDR, COL (no funcionarios) y CEU (categoría a extinguir).

Es innegable que la situación ha cambiado desde aquel 7 de julio de 1981 cuando se publicó en el BOE (BOE, 1981) el nombramiento M. ${ }^{a}$ Dolores Climent Morato, primera catedrática de la UPV. Sin embargo, más de tres décadas después sólo un 16\% de los CU de la UPV son mujeres, limitándose su presencia al 55\% de los departamentos, mientras que un 93\% de los departamentos tiene CU hombres.

Este fenómeno refleja la existencia de un «techo de cristal» para las mujeres, con consecuencias en cuanto al grado de estabilidad laboral, niveles de ingresos, control y poder de decisión, asociados a las distintas categorías laborales.

Explorar las causas que han llevado a esta situación de desigualdad en la UPV no es objetivo de este trabajo, pero seguramente coinciden con las de otras instituciones de Educación Superior. En la Universidad actual, a pesar de que formal y aparentemente todos sus miembros están sometidos a un sistema meritocrático, persisten la opacidad de los sistemas de selección y promoción y el sesgo de género en la evaluación de méritos (Castaño; Pastor y Acosta). Además, a estas barreras se añaden las conocidas dificultades para conciliar trabajo y vida personal (Castaño) porque las mujeres siguen responsabilizándose de la mayor parte de la carga reproductiva y de las labores de cuidado (Guil 2016). En consecuencia, la Universidad no puede considerarse una institución 
igualitaria, porque todavía presenta obstáculos invisibles de tipo político, de intereses, de prejuicios, etc. que perjudican y discriminan indirectamente a las mujeres. Pero lo más grave es que estas desigualdades de género tienden a negarse, son inconscientes y, bajo el espejismo de la igualdad formal, se consideran ya resueltas. De ahí la importancia de este diagnóstico como paso previo para favorecer el debate de la comunidad universitaria y para empezar a trabajar en la resolución del problema.

El escaso compromiso político de la UPV como institución, que se ha limitado al mero cumplimiento de las leyes, ha sido determinante de la lentitud del cambio. Un aumento claro de su compromiso con las políticas de igualdad, plasmado a través del apoyo incondicional a su unidad de igualdad, se revela como medida indispensable para la reforma de su cultura institucional y para transformarse en una institución más inclusiva. A partir de ahí medidas concretas podrían dirigirse a evitar sesgos en los procesos de selección y promoción a través de, por ejemplo, formación en conciencia de género para las comisiones de contratación y aumento del porcentaje de mujeres participantes en las mismas, y la consideración de los permisos de maternidad en la valoración de méritos y del currículum. Asimismo, para las escuelas o departamentos con peores cifras de participación femenina podría llevarse a cabo una discriminación positiva temporal, hasta conseguir cifras más equilibradas. A todo ello se sumaría la promoción de prácticas que faciliten la conciliación de hombres y mujeres.

La UPV presume de ocupar puestos destacados en los principales rankings nacionales e internacionales de Educación Superior. Sin embargo, en términos de igualdad se sitúa casi a la cola de las universidades españolas. Aunque su especialización en ingeniería y tecnología, campos tradicionalmente masculinos, supone un desafío adicional, ha llegado el momento de promover la equidad, eliminando barreras estructurales para aumentar la participación de las mujeres, con una perspectiva a largo plazo. No actuar supone mermar la capacidad de innovación y competitividad de la propia institución, desaprovechar parte de la inversión social e individual en capital humano, además de ir contra la justicia social, ¿puede permitírselo?

\section{REFERENCIAS BIBLIOGRÁFICAS}

Acosta, Ana, Inma Pastor y Angel Belzunegui. «Las políticas de igualdad en las universidades españolas: aproximación desde un estudio de redes». 1st International Virtual SBRLab Conference. "Finding solutions for a post-crisis society», Universitat Rovira i Virgili, Tarragona (2015): 291-307. 24-05-2016. 
Alcañiz, Mercedes (ed.). «La conciliación de la vida laboral y familiar en la Universitat Jaume I». Publicacions de la Universitat Jaume I, Servei de Comunicació i Publicacion, 2014. 15-01-2016

Alonso, Maria José. «Las académicas. Profesorado universitario y género». Revista de Educación, 328 (2002): 465-475.

Anguita, Rocio. (coord.). Las mujeres en la Universidad de Valladolid. Universidad de Valladolid, 2003.

Antón, Susana. Informe: Académicas en la Universidad de Alicante. Centro de Estudios sobre la Mujer-Universitat d'Alacant, 2005. 16-10-2015.

Barberá, Ester, Maria Josefa Lafuente y Maite Sarrió. La promoción profesional de las mujeres en la Universidad. Promolibro, Valencia, 1998.

Basset-Salom, Luisa; Arianna Guardiola-Víllora y Begoña Serrano-Lanzarote. «20 años de presencia femenina en la Escuela Técnica Superior de Arquitectura de Valencia y en el Departamento de Estructuras». Jornadas Internacionales de Arquitectura y Urbanismo desde la perspectiva de las arquitectas. Instituto Juan de Herrera (2009).

BOE n. ${ }^{\circ} 232$ del 29 de septiembre de 1981. 20-01-2016

Castaño, Cecilia. «La nueva gestión pública y las políticas de igualdad de género en las universidades». Investigaciones Feministas 7.2 (2016): 225-245.

Comission of the European Communities. Progress towards Lisbon objectives in education and training. SEC. Bruselas, 2005.

De los Cobos, Francisco. «Planes de igualdad en las universidades españolas. Análisis de contenido desde las ciencias sociales». Barataria: Revista CastellanoManchega de Ciencias Sociales 14 (2012): 117-130.

Elizondo, Arantxa, Ainhoa Novo y María Silvestre. «La presencia de mujeres y hombres en las universidades españolas». Aequalitas: Revista Jurídica de Igualdad de Oportunidades entre Mujeres y Hombres 21 (2007): 25-34.

Elizondo, Arantxa, Ainhoa Novo y María Silvestre. Igualdad de mujeres y hombres en las universidades españolas, Universidad del País Vasco, Facultad de Ciencias Sociales y de la Comunicación, Departamento de Ciencia Política y de la Administración. Instituto de la Mujer, 2010.

Escolano, Esther. «Mujeres y función pública. El personal de administración y servicios de la Universitat de València», Publicacions de la Universitat de València Valencia, 2002.

Escolano, Esther. «Entre la discriminación y el mérito: las profesoras en las universidades valencianas». Publicacions de la Universitat de València, València, 2006.

Estellés-Miguel, Sofía, Antonio Navarro-García, Marta E. Palmer Gato y José Miguel Albarracín Guillem. «Gender Inequality in University Administration and Services», Business and Management Research 3.4 (2014): 89-95.

European Commission «Structural Changes in Research Institutions. Final report» Science in Society (2012). 
European Commission. She figures, 2015: Gender in Research and Innovation. Directorate General for Research and Innovation. Brussels, 2016. 21-04-2016 Fernández-Coronado, Rosario y $\mathrm{M}^{\mathrm{a}}$ Eugenia González. Mujeres y hombres en la Universitat de València, Unitatd'Igualtat - Universitat de València, 2009.

Guil, Ana. «Techos de Cristal en la Universidad Hispalense». Informe final del proyecto del Plan Nacional I+D+i 2001-2004 Sevilla, Universidad de Sevilla, 2005. 15-01-2016.

Guil, Ana. «Mujeres y Ciencia: Techos de Cristal». ECCOS Revista de Investigación Científica, Universidade Nove de Julho, Brasil. www.uninove.br/ publicaçoês, (2008): 213-232.

Guil, Ana y Consuelo Flecha.»Universitarias en España: de los inicios a la actualidad». Revista Historia de la Educación Latinoamericana 17.24 (2015): 125-148.

Guil, Ana. «Techos universitarios de cristal blindado». Investigaciones Feministas 7.2 (2016): 25-39.

Guardiola-Víllora, Arianna, Luisa Basset-Salom y Elena Navarro-Astor. «Women's participation in academic management teams: the case of the Universitat Politècnica de València». Proceedings of INTED, (2016): 1880-1887.

Instituto Nacional de Estadística, INE. Estadística para la Enseñanza Universitaria en España, curso 2004-2005: Personal docente de los centros propios de las Universidades Públicas por Área de conocimiento, Sexo y Categoría, 2006. < http://www.ine.es/> consultado el 16-10-2015

Izquierdo, M. ${ }^{a}$ Jesús (dir.). El sexisme a la UAB. Propostes d'actuació i dades per a un diagnòstic. Barcelona, GESES-Universita Autònoma de Barcelona, 2004.

Jiménez, Marta Esther. «El género en el profesorado. El caso de las profesoras universitarias». Clepsydra. Revista de Estudios de Género y Teoría Feminista 2 (2003): 71-101.

Lecuona, María del Pino. «Género y Universidad». Enseñanza E Teaching 23 (2005): 143-160.

Lozano Cabezas, Inés, Marcos Jesús Iglesias Martínez y Ma Ángeles Martínez Ruíz. «La cultura de igualdad de oportunidades en el tejido universitario europeo». Revista Interamericana de Investigación, Educación y Pedagogía 6.2 (2013): 31-59.

Lozano Cabezas, Inés y Marcos Jesús Iglesias Martínez. «La presencia de académicas a lo largo de la historia en la Educación Superior española». Journal for Educators, Teachers and Trainers 5.3 (2014): 204-216.

Lozano Cabezas, Inés, Marcos Jesús Iglesias Martínez y Mª Ángeles Martínez Ruíz. «Un estudio cualitativo sobre los diferenciales de género en la educación superior: percepciones de las académicas en contextos masculinizados». La Manzana de la Discordia 11.1 (2016): 41-54.

Marqués, Marisol, M. Nuria Salán y Silvia Gómez. Estudio comparativo de la carrera académica del profesorado en las universidades públicas politécnicas españolas 
desde una perspectiva de género. Universitat Politècnica de Catalunya, 2012. 16-10-2015.

Matus-López, Mauricio y Nazareth Gallego-Morón. «Techo de cristal en la Universidad. Si no lo veo no lo creo». Revista Complutense de Educación 26.3 (2015): 611-626.

Mauleón, Elba y María Bordons. «Indicadores de actividad tecnológica por género en España a través del estudio de patentes europeas». Revista Española de Documentación Científica, 37(2):e043. (2014). DOI: http://dx.doi. org/10.3989/ redc.2014.2.1093

MEC-UMYC. Científicas en cifras 2013. Estadísticas e indicadores de la (des)igualdad de género en la formación y profesión investigadora. Ministerio de Economía y Competitividad. Unidad de Mujeres y Ciencia, 2014.

MECyD. Datos y cifras del Sistema Universitario Español. Curso 2014/2015. Ministerio de Educación, Cultura y Deporte (2015a). <http://www.mecd. gob.es/dms/mecd/educacion-mecd/areas-educacion/universidades/estadisticas-informes/datos-cifras/Datos-y-Cifras-del-SUE-Curso-2014-2015.pdf>, consultado el 16-10-2015.

MECyD. Avance de la Estadística de estudiantes universitarios. Curso 20142015. Cap III. Datos por Universidad. Ministerio de Educación, Cultura y Deporte (2015b). <https://www.educacion.gob.es/educabase/menu. do? type $=$ pcaxis\&path=/Universitaria/Alumnado/Avance/2014-2015/ CapituloIII/Publicas\&file=pcaxis\&l=s0>, consultado el 16-10-2015.

Morales, M. ${ }^{a}$ Jesús, M. José Luna y Ana Isabel Esteban. «Diagnóstico de paridad en la Universidad: análisis a través de indicadores». Revista de Universidad y Sociedad del Conocimiento 7.2 (2010): 1-14.

Navarro-Astor, Elena, Marisa Román-Onsalo y Margarita Infante-Perea. «Revisión internacional de estudios de barreras de carrera bajo la perspectiva de género en la industria de la construcción». Innovar 26.61 (2016): 103-118.

Pastor, Inma, Paloma Pontón, Angel Belzunegui y Nuria Serret, «Políticas de igualdad en la Universidad: una reflexión sobre los planes de igualdad en América Latina y España». Proceedings V Congreso Universitario Internacional «Investigación y Género», SIEMUS, Universidad de Sevilla (2014): 639-658

Pastor, Inma, Paloma Pontón, Angel Belzunegui y Ana Acosta. «Gender Policies in Spanish Universities: From Regulation to Equality Plans». En: Desivilya H. y Costea, CE (editores): Women's Voices in Management. Identifying Innovative and Responsible Solutions. Palgrave Macmillan (2015): 34-51.

Pastor, Inma y Ana Acosta. «La institucionalización de las políticas de igualdad de género en la Universidad Española. Avances y retos». Investigaciones Feministas 7.2 (2016): 247-271.

Pastor-Gosálbez, Inma, Glòria Solsona, y Francesc Valls. «Desigualtat a la universitat per raó de gènere? La situació de les dones a la Universitat Rovira i 
Virgili: de la diagnosi a les mesures d'igualtat». Revista Catalana de Sociologia 25 (2010): 55-72.

Perelló, Fátima. Asimetrías de género en la Universitat de València. Universitat de València, 2012.

Perelló, Fátima «La vulnerabilidad profesional femenina a la vuelta de la esquina. El caso del personal de administración y servicios de la Universitat de València». Arxius 28.9 (2013): 3-104.

Sánchez de Madariaga, Inés. «Advancing Gender Equality in Research and Innovation in Europe and beyond: COST network genderSTE», Journal of Research in Gender Studies 3 (2013): 131-143.

Sánchez de Madariaga, Inés. Women at UPM. Gender statistics at Universidad Politécnica de Madrid. UPM. (2014). 16-10-2015.

Santamarina, M. Pilar, Ma Rosa Cerdá, Amparo Quilis y Ana Muñoz. Antecedentes y acciones emprendidas en la UPV en materia de igualdad. Vicerrectorado de Asuntos Sociales y Responsabilidad Social Corporativa. UPV, 2011.

Santos, Juan Antonio, David Muñoz-Rodríguez y Manuela Poveda, «En cuerpo y alma. Intensificación y precariedad en las condiciones de trabajo del profesorado universitario». Arxius de Sociología, 32 (2015): 13-44.

Santos, José Luis. Nacimiento de una Universidad (algunos recuerdos). Servicio de Publicaciones UPV. Valencia. Universitat Politècnica de València, 1993. 